\title{
The Effects of an Exercise and Kinesiotape Intervention on Forward HeadRounded Shoulder Posture and Scapular Dyskinesis
}

Lucas Klawiter

Follow this and additional works at: https://researchrepository.wvu.edu/etd

\section{Recommended Citation}

Klawiter, Lucas, "The Effects of an Exercise and Kinesiotape Intervention on Forward HeadRounded Shoulder Posture and Scapular Dyskinesis" (2017). Graduate Theses, Dissertations, and Problem Reports. 5988.

https://researchrepository.wvu.edu/etd/5988

This Thesis is protected by copyright and/or related rights. It has been brought to you by the The Research Repository @WVU with permission from the rights-holder(s). You are free to use this Thesis in any way that is permitted by the copyright and related rights legislation that applies to your use. For other uses you must obtain permission from the rights-holder(s) directly, unless additional rights are indicated by a Creative Commons license in the record and/ or on the work itself. This Thesis has been accepted for inclusion in WVU Graduate Theses, Dissertations, and Problem Reports collection by an authorized administrator of The Research Repository @ WVU. For more information, please contact researchrepository@mail.wvu.edu. 
The Effects of an Exercise and Kinesiotape Intervention on Forward Head/Rounded Shoulder Posture and Scapular Dyskinesis

Luke Klawiter, ATC, CES

Thesis submitted to the College of Physical Activity and Sports Sciences at West Virginia University

in partial fulfillment-of the requirements for the degree of

Master of Science in Athletic Training

\author{
Michelle Sandrey, PhD, ATC, Chair \\ Jean McCrory, $\mathrm{PhD}$ \\ Jordan Feathers, MPT, OCS, CSCS
}

Department of Sport Sciences

Morgantown, West Virginia

2017

Keywords: Forward Head Posture, Rounded Shoulder Posture, Scapular Dyskinesis, Kinesiotape, Treatment 


\begin{abstract}
The Effects of an Exercise and Kinesiotape Intervention on Forward Head/Rounded Shoulder Posture and Scapular Dyskinesis

Luke Klawiter, ATC, CES
\end{abstract}

Context: Forward head and rounded shoulder posture as well as scapular dyskinesis are forms of improper posture that have been linked to neck and shoulder pain. Treatment for forward head posture (FHP), rounded shoulder posture (RSP), and scapular dyskinesis has consisted of an exercise protocol. Kinesiotape (KT) has recently been used as a treatment method but there is a lack of research on the effectiveness, or whether exercise or KT is better than the other.

Objective: The objective of the study was to compare a KT intervention to a strengthening and stretching program for correction of FHP, RSP, and scapular dyskinesis in a healthy, nonathletic, college age population. Design: The study was a repeated measures pre/post-test design. Setting: The testing and interventions took place at an athletic training research lab at a DI mid-Atlantic university. Only one clinician administered the testing and interventions.

Patients and Other Participants: The study included 20 healthy college students that are premajor and major athletic training students. There were 10 subjects ( 7 females, 3 males, $20.30 \pm .82 \mathrm{yrs}, 171.07 \pm 11.82 \mathrm{~cm}, 79.47 \pm 13.79 \mathrm{~kg}$ ) in the exercise group and 10 subjects $(7$ females, 3 males, $20.40 \pm 1.43 \mathrm{yrs}, 166.61 \pm 11.99 \mathrm{~cm}, 69.40 \pm 11.48 \mathrm{~kg}$ ) in the kinesiotape group. Inclusion criteria for the study contained those subjects who were healthy with no history of upper extremity injury or surgery within six months leading up to the study and students with status as pre-major or major students in the undergraduate athletic training program and were classified as having FHP, RSP, and/or scapular dyskinesis. Exclusion criteria for the study contained those subjects currently engaged in a glenohumeral joint or cervical training program, current glenohumeral joint, neck, arm hand, or back injuries, or surgery involving the glenohumeral joint, arm, hand, back, or neck within six months of the study. Interventions: There were two intervention groups undergoing a four-week program. One group participated in a strengthening and stretching exercise protocol, while the other group had KT applied to the upper back and shoulders for a duration of five days with two days of no tape in a seven-day period. Main Outcome Measures: The dependent variables measured were forward head posture as assessed by the craniovertebral angle (CVA) in degrees, rounded shoulder posture as assessed by the forward shoulder angle (FSA) in degrees, and scapular dyskinesis as assessed using scapular dyskinesis scoring (0-3) for each scapula. Results: Negative, weak, correlations with high p-values were found between cell phone use and CVA, RSA, and scapular dyskinesis score as well as between computer use and CVA and scapular dyskinesis score, while a positive, weak correlation with a high p-value between computer use and RSA. There was no statistically significant group, time, or group $\mathrm{x}$ time interaction for the CVA and RSA measurements. There was a statistically significant time main effect for the scapular dyskinesis score $(\mathrm{F}=12.570$, $\mathrm{p}<.01$ ) but not for group or group $\mathrm{x}$ time interaction. MDC was achieved for the CVA and scapular dyskinesis score for both groups. Conclusions: Both groups improved pre/post-test for scapular dyskinesis. No other results were significant for CVA and RSA. In addition, there was a moderate inverse relationship between cellphone/computer use and FHRSP and scapular dyskinesis. Due to the results of the group mean differences and MDC for the CVA and RSA, further research should be conducted. 


\section{ACKNOWLEGDEMENTS}

I would like to start by thanking my Lord and Savior, Jesus Christ. Without Him and the sacrifice He made none of this would have been possible.

Next I would like to thank my mom. She has been my rock ever since she brought me into this world. I know being a single mother was never easy, and I will forever be grateful for you putting me through college as well as the lessons and virtues you have taught me that I still hold to this day. I love you so very much.

I would also like to thank my grandparents, Jack and Joyce, who you are no longer here today. They played a vital role in my development not only as a student, but more importantly, the man I am today. Both of you will always be in my heart.

I would like to thank my family overall. I have not been able to be very active in our family for these past two years but your support has been never failing. Thank you for supporting my dreams and encouraging me along the way.

Thank you to my committee members, Dr. Jean McCrory and Jordan Feathers. Thank you for all of your time, patience, and effort in this process.

A huge thanks to my committee chair and graduate advisor, Dr. Michelle Sandrey. All of your hours reading my drafts and pushing me to my limit did not go unnoticed. You kept me on my toes for the past two year and for that I am thankful.

To my clinical supervisor at HealthWorks, Mike Casselman, thank you for giving me the opportunity to grow clinically and personally and aiding me along the way these past two years.

To my subjects. You guys rocked. I enjoyed getting to know each and every one of you during my study and I am very appreciative for all of your commitment. I wish you all the best of luck in your future endeavors.

I would like to give a shout out to my two cats, Dusty and Thad, for providing me with stress relief during this process.

Lastly, I want to thank everyone else I did not mention that has helped me get to this point. You each have played a significant role in my life and I am incredibly appreciative. 


\section{TABLE OF CONTENTS}

ACKNOWLEGMENTS......................................................................

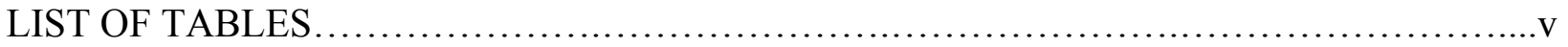

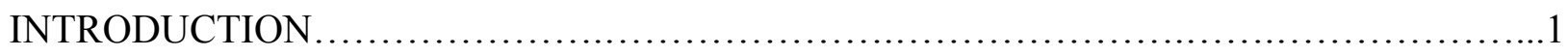

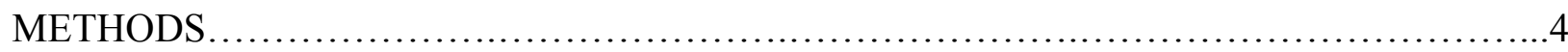

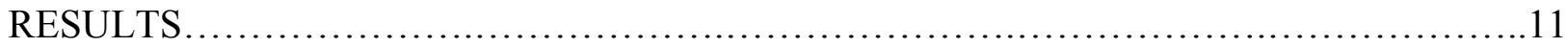

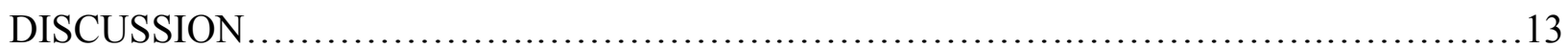

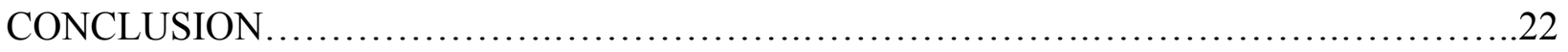

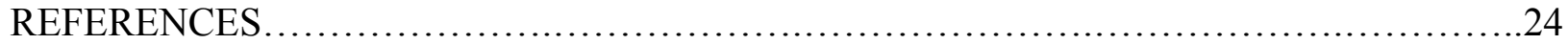

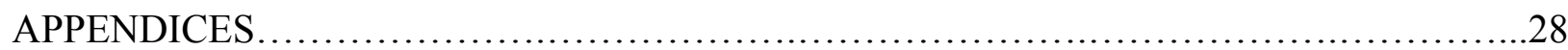

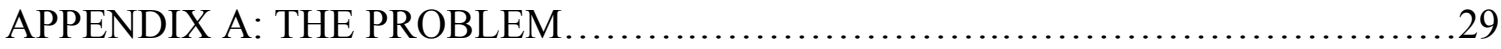

APPENDIX B: LITERATURE REVIEW ............................................38

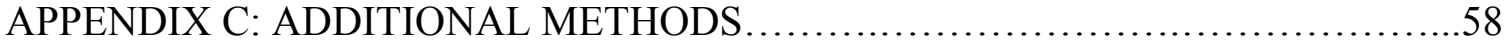

APPENDIX D: ADDITIONAL RESULTS.............................................74

APPENDIX E: RECOMMDENDATIONS FOR FUTURE RESEARCH................76

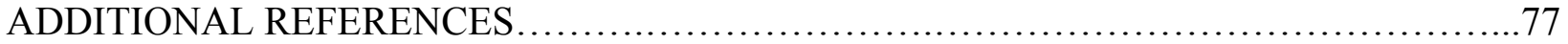




\section{LIST OF TABLES}

Table B1. Measurement Techniques for FHRSP and Scapular Dyskinesis...................46

Table B2. Treatment Techniques for FHRSP and Scapular Dyskinesis......................53

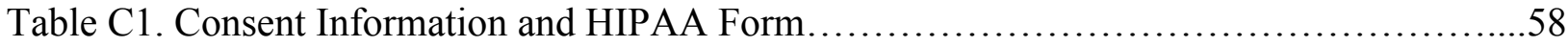

Table C2. Subject Questionnaire ................................................62

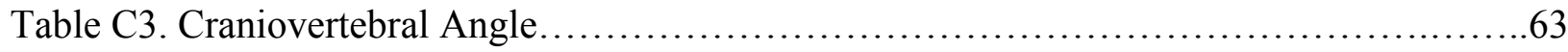

Table C4. Shoulder Angle.......................................................63

Table C5. Scapular Dyskinesis Measurement......................................64

Table C6. Data Collection Sheet.....................................................65

Table C7. Exercise Intervention Protocol...........................................66

Table C8. Strengthening and Stretching Intervention Exercises.........................67

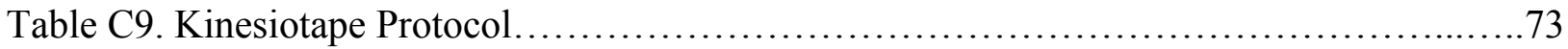

Table D1. Baseline Group Measurements for CVA, RSA, and Scapular Dyskinesis Score.........74

Table D2. Pre-test/Post-test Measurements for CVA, RSA, and Scapular Dyskinesis Score.......74

Table D3. Mean Time Difference \& MDC Values for Main Outcome Measures.......................74

Table D4. Time Effect for CVA, RSA, and Scapular Dyskinesis Score.............................75

Table D5. Group Effect for CVA, RSA, and Scapular Dyskinesis Score...........................75 


\section{INTRODUCTION}

Neck and shoulder pain is becoming very prevalent around the world and is present in all populations. Studies have shown that the prevalence of neck pain in the general population may range from $.4 \%-86.8 \%,{ }^{1} 30 \%-50 \%,{ }^{2}$ or $31 \%{ }^{3}$ depending on the source. Shoulder pain has been found to occur in the population at rates of $6.7 \%$ to $66.7 \%{ }^{4}$ or $10 \%-57.9 \% .{ }^{5}$ Neck and shoulder pain has also shown a high prevalence of comorbidity within the general population as well. ${ }^{3,6-8}$ While pain is typically always an annoyance, neck and shoulder pain specifically has limited both life and work activities. ${ }^{1}$ The possible risk factors and causes of neck and shoulder pain include previous injury, lack of physical activity, repetitive activities, ${ }^{7,9}$ use of computer, cellphones, ${ }^{10,11}$ and tablets, ${ }^{10}$ and posture. ${ }^{1,8,12}$ Of possible risk factors and causes, some are modifiable and can be altered to decrease the pain and treat the underlying pathology, while others may not.

Posture has been linked to shoulder and neck pain by multiple studies. ${ }^{6,13-16}$ Specific postural distortions include forward head posture (FHP) and rounded posture (RSP) ${ }^{14,16}$ FHP is when the head protrudes anteriorly due to the upper cervical vertebrae being placed into extension and the lower cervical vertebrae into flexion for a prolonged period of time. ${ }^{16}$ The unnatural displacement of the cervical vertebrae places stress onto the cervical neck flexor and extensor muscles as well as the cervical intervertebral disks. ${ }^{16}$ FHP has been increasing in prevalence due to an increase in technology use and repetitive activities in the industrial and athletic settings. ${ }^{9-11,17,18}$ The tight anterior chest muscles and a weakened posterior thoracic muscles lead to RSP as the glenohumeral joint shifts anteriorly. ${ }^{19,20}$ The anterior shift in the glenohumeral joint also occurs with the scapula, distorting the scapulohumeral rhythm. ${ }^{21}$ The 
repetitive activities constantly overwork and cause a tightening of the pectoralis minor, sternocleidomastoid, and the upper trapezius, which may cause FHP and RSP. ${ }^{16,19,22}$

A change in scapulohumeral rhythm is known as scapular dyskinesis and further SICK scapula. SICK is an acronym for scapular malposition, inferior medial border prominence, coracoid pain and malposition, and dyskinesia of scapular movement. ${ }^{23-26}$ Scapular dyskinesis, along with FHP and RSP, has been linked to pain caused by a postural distortion. ${ }^{16,23,27,28}$ The abnormal positioning of the scapula, glenohumeral joint, and cervical vertebrae change the force couples and length-tension relationships associated with those structures and cause pain. ${ }^{16,23}$

The recommended treatment for FHP, RSP, and Scapular dyskinesis has typically involved exercises that focus on the strengthening of weak or inactive muscles and stretching of tight or overactive muscles..$^{23,29-31}$ The specific muscles for FHP that are strengthened are the deep cervical flexors and the muscles stretched are the upper trapezius (UT) and sternocleidomastoid. ${ }^{16,29,30}$ For RSP, muscles to be strengthened include the lower trapezius (LT) and serratus anterior (SA), while stretching the pectoralis minor (PM). ${ }^{19,29,31}$ Due to the alteration in position of the scapula and the glenohumeral joint as well as the alteration in force couples above and below the scapula, ${ }^{27,32}$ exercises to correct scapular dyskinesis are similar to the ones included for the treatment of FHP and RSP. In addition to the deep cervical flexors, LT, and SA, the scapula stabilizers should be strengthened while the posterior glenohumeral joint capsule, UT and PM are stretched..$^{23,27,32}$ Despite the recommended treatment protocol, mixed results on the effectiveness of a strengthening and stretching program for correcting FHP, RSP, and scapular dyskinesis is evident in the literature. Studies by Ruivo, ${ }^{29}$ Gupta, ${ }^{30}$ Burkhart, ${ }^{23}$ and Lee ${ }^{19}$ have indicated that an exercise protocol was effective in decreasing FHP ${ }^{29,30}{ }^{\mathrm{RSP}},{ }^{19}$ and scapular 
dyskinesis, ${ }^{23}$ whereas Wong $^{31}$ noted no significant difference between the intervention and placebo group for correcting RSP.

While studies ${ }^{16,19,23,27,29-32}$ have shown the effectiveness or lack of effectiveness of a strengthening and stretching exercise protocol for the correction of FHP, RSP, and scapular dyskinesis, those protocols are very time consuming for such chronic diseases and have limited effectiveness leading to non-compliance. Lee et al. ${ }^{19}$ has shown potential in the use of a shoulder brace as an effective means to correct the postural distortions over time. However, the findings were limited and additional research has suggested that kinesiotape, an elastic adhesive tape, may be able to stimulate sensory nerves in the form of proprioception, just as with the shoulder brace. ${ }^{33}$ Kinesiotape is a relatively new treatment method and thus the research is limited as well. ${ }^{34}$ The indications for kinesiotape are for neuromuscular re-education, inflammation and swelling reduction, promoting circulation, and proprioception as well as one case of decreasing RSP in a sedentary worker. ${ }^{34-36}$

Neck and shoulder pain has been shown to be a medical hindrance in the general population as well industrial and athletic populations. ${ }^{3-8}$ An increase in technology use (computers, smart phones, tablets) has led to a forward and flexed posture and further an increase in neck and shoulder pain in a younger population. ${ }^{10-12}$ Specific and common postural distortions include FHP, RSP, and scapular dyskinesis. ${ }^{6,13-16}$ These postures change the alignment of anatomical structures and thus alter force couples and the biomechanics of the body. ${ }^{6,13-16}$ Treatment for FHP, RSP, and scapular dyskinesis has typically been isolated to a strengthening and stretching exercise protocol. ${ }^{19,23,29-32}$ However, kinesiotape has shown to be a limited but effective means of correcting postural distortions. ${ }^{34-36}$ Most literature on the correction of FHP, RSP, and scapular dyskinesis has not focused on a college-age population, but rather on the 
industrial population. This is important as younger patients $(<50$ years old) have been found to have FHP more frequently and thus have a statistically significant increase in neck pain. ${ }^{16}$ In addition, the literature has focused on a strengthening and stretching protocol as a treatment method for FHP, RSP, and scapular dyskinesis, and has not examined the uses and effectiveness of KT as a treatment. Further, as studies on exercise and kinesiotape have occurred in isolation, few studies have evaluated and compared the individual effectiveness. Therefore, the purpose of this study is to compare a kinesiotape intervention to a strengthening and stretching program for correction of FHP, RSP, and scapular dyskinesis in a healthy, non-athletic, college age population. A secondary purpose of this study is to evaluate the relationship between FHP, RSP, and scapular dyskinesis and the extent of cell phone and computer use.

\section{METHODS}

Design

The study was a repeated measures design, with pre and post-testing. The subjects were tested before the beginning of the study and then four weeks later at the conclusion of the study. The independent variables were the group the subject was be assigned to (exercise group or kinesiotape group) and time (pre and post-test). The dependent variables were the measurements of the craniovertebral angle (CVA) and forward shoulder angle (FSA) and the scoring of scapular dyskinesis for each scapula, 0-3, for a combined maximum score of 6 . Subjects

Twenty subjects were recruited for the study using a convenience sample of healthy college-aged students that are pre-major and major athletic training students. All twenty subjects completed both testing periods (baseline and follow-up) as well as the four weeks of one of the interventions. There were 10 subjects ( 7 females, 3 males, $20.30 \pm .82$ yrs., $171.07 \pm 11.82 \mathrm{~cm}$, 
$79.47 \pm 13.79 \mathrm{~kg}$ ) in the exercise group and 10 subjects ( 7 females, 3 males, $20.40 \pm 1.43 \mathrm{yrs}$., $166.61 \pm 11.99 \mathrm{~cm}, 69.40 \pm 11.48 \mathrm{~kg}$ ) in the kinesiotape group. Before data collection, a questionnaire was given to each subject to determine demographic information, activity level, and injury history to determine eligibility for the study. Inclusion criteria were those subjects who were healthy with no history of upper extremity injury or surgery within six months leading up to the study and students with status as pre-major or major students in the undergraduate athletic training program. Subjects were classified as having FHP, RSP, and/or scapular dyskinesis based on a CVA less than 50 degrees, a FSA less than 52 degrees, and a combined scapular dyskinesis score less than 6, respectively. Exclusion criteria were those subjects currently engaged in a glenohumeral joint or cervical training program, current glenohumeral joint, neck, arm hand, or back injuries, or surgery involving the glenohumeral joint, arm, hand, back, or neck within six months of the study. All twenty subjects completed both testing periods (baseline and follow-up) as well as the four weeks for one of the interventions. This study was approved by the Office of Research Compliance at the Institution.

Instruments

Craniovertebral Angle (CVA): The CV angle is an objective measure for forward head posture that has been found to have success with test-retest and has shown that decreased CV angles correlate with increased cervical pain as well as a greater degree of forward head posture. ${ }^{37}$ The $\mathrm{CV}$ angle measures the angle between a horizontal line through the body of the $7^{\text {th }}$ cervical vertebra and a line from the $7^{\text {th }}$ cervical vertebra and the tragus of the ear. ${ }^{14} \mathrm{~A} 50$-degree angle has been used in research as a reference angle; an individual with less than a 50-degree CV angle is considered to have a FHP. ${ }^{29}$ This method of measurement for CVA has been shown to have good to very good reliability, with a .78 interrater reliability. ${ }^{29}$ The study by Lau ${ }^{37}$ 
determined there was high intra-rater reliability (ICC .86-.94) and interrater reliability (.85-.91) when measuring the CVA.

Forward Shoulder Angle (FSA): This method of measurement for FSA has shown to have good reliability and very good interrater reliability (.66). ${ }^{29}$ Rounded Shoulder assessment is the use of the angle between a line from the $7^{\text {th }}$ cervical spinous process to the acromion process and a horizontal line through the acromion process - the shoulder angle. A 52-degree reference angle has been used; an individual with less than a 52-degree shoulder angle are classified as having a rounded shoulder posture. ${ }^{29}$

Scapular Dyskinesis Scoring: The scoring system used ${ }^{38}$ is a variation of the subjective classification system used by McClure. ${ }^{24}$ The study by McClure ${ }^{24}$ determined there was a 75$82 \%$ agreement between raters when scapular dyskinesis is classified as either normal, subtle, or present. Butowicz ${ }^{38}$ adapted the classification system by giving a numerical value for scapular motion. Pain during movement was scored as a 0 , obvious scapular winging during movement was scored as a 1, subtle winging scored a 2, and normal scapular motion scored a 3 . This was completed for both scapulae for a maximum combined score of $6 .^{38}$

Procedures

The main researcher approached each prospective and curriculum athletic training class at a Mid-Atlantic University to present the research idea and to ask for volunteers. Those subjects that met all inclusion criteria and completed the informed consent form with HIPAA included (Table $\mathrm{C} 1$ ) and subject questionnaire (Table C2), were invited to volunteer to participate in the study. During the baseline testing, each subject was assigned a subject number in which they were referred to for the duration of the study. The subjects were then randomly assigned into one of two groups: exercise or kinesiotape with 10 subjects in each group. Prior to 
the testing period, times were established for subjects in the exercise and kinesiotape groups to meet with the researcher once a week, for four weeks, for approximately 10 minutes per session. Pre-testing procedures included measurement of the craniovertebral angle and forward head angle and scoring of scapular dyskinesis. Subjects had still photograph taken while wearing reflective markers that was used later with the Hudl app (Agile Sports Technologies, Inc., Lincoln, Nebraska) for analysis of the CVA and FSA and a video was taken of each subjects' scapular movement during weighted arm abduction and flexion for scapular dyskinesis scoring.

Following baseline testing and random group assignment, each week, for four weeks, the subjects in the exercise and kinesiotape groups met at a predetermined time with the researcher. During the first predetermined meeting the exercise group was educated on a set of exercises referred to as the home exercise plan (HEP) and given a picture copy of each exercise with instructions for completion as well as predetermined sets and repetitions. The exercise group completed the HEP on their own at a predetermined time period daily for the duration of the study. The subjects in the exercise group also completed a series of exercises separate from the HEP with the main researcher during the weekly meeting. The subjects in the kinesiotape group had kinesiotape applied to the back and shoulders in a predetermined pattern. The subjects kept the tape on for a period of five days and then removed the tape for two days during a seven-day period. The subjects then had kinesiotape reapplied and repeated this process at the beginning of each of the four weeks of the intervention period.

At the conclusion of the four weeks, each subject met with the researcher to have followup measurements taken. The same procedures used during the baseline measurements were again used for the CVA, FHA, and scapular dyskinesis scoring. Markers for the location of the 
patient during measurement were placed on the floor of the testing room to ensure consistency in angle measurements, and will be five feet from the researcher holding the iPad, (Table C3-4). Pre-test/Post-test

Pre-test procedures were completed prior to the start of the intervention programs. Posttesting procedures were conducted within the week after the conclusion of the four-week intervention programs. The post-testing procedures were the same as the pre-testing procedures. Data from the pre-test/post-test was then collected by the researcher and was analyzed to compare the pre-test data and the post-test data.

Craniovertebral Angle (CVA): ${ }^{29,37,39}$ Each subject had reflective markers placed on the spinous process of the $\mathrm{C} 7$ vertebrae and the tragus of the ear on one side of the subject's body (Table C3-4). A still photograph was taken using an iPad from a sagittal view with the reflective markers facing the iPad (Table C3-4).

Forward Shoulder Angle (FSA): ${ }^{19,29,39}$ Each subject had reflective markers placed on the spinous process of the $\mathrm{C} 7$ vertebrae and the acromion process of the shoulder on the same side of the body as the tragus (Table C3-4). A still photograph was then taken using an iPad from a sagittal view with the reflective markers facing the iPad (Table C3-4).

Scapular Dyskinesis Scoring: ${ }^{24,38}$ Each subject stood in a relaxed position, with the shirt removed if a male subject and a sports bra worn for female subjects. The subjects performed five repetitions of bilateral shoulder flexion and abduction with a $2 \mathrm{lb}$ weight. (Table C5) This procedure was followed for both the right and left sides simultaneously. An iPad was used to record the subject during examination that was then analyzed and scored at a later time. (Table C6)

Intervention 
The exercises selected for the exercise intervention protocol were based on the previous literature ${ }^{19,23,29-31}$ on the correction of forward head/rounded shoulder posture (FHRSP) and scapular dyskinesis. The HEP (Table C7A) for the exercise intervention group consisted of strengthening and stretching exercises for the glenohumeral joint, neck, and scapular stabilizers. The strengthening exercises included chin tucks (2 sets of 10; $5 \mathrm{sec}$. hold), Y's to I's (3 sets of 10 ), wall washes (3 sets of 10), upright scapula punches (3 sets of 10), corner stretch (3 sets 30 sec. hold), and a static sternocleidomastoid (SCM) stretch (3 sets 30 sec. hold for each side). (Table C7A) The HEP was taught and practiced during the baseline session; subjects continued the at home exercises once daily for four weeks. The weekly session with each subject consisted of the researcher assisting the subject with additional exercises. Those additional exercises included scapular PNF (1 set to fatigue for each diagonal), Y's to I's with ball chin tuck (3 sets of 10), and passive SCM and pectoralis minor stretching (3 sets $30 \mathrm{sec}$. hold for each side). Refer to Tables C7B and C8.

The subjects assigned to the kinesiotape group provided proper instructions while wearing the kinesiotape. The pattern selected (Table C9) had the subject retract both scapulae while the kinesiotape was placed in a diagonal. One strip started from the patients' superior border of the right scapula to the inferior border of the left scapula while the other strip started from the superior border of the left scapula to the inferior border of the right scapula. The kinesiotape acted as a proprioceptive reminder for the subject to keep both scapulae retracted to maintain proper posture. The subjects were instructed that when they felt the tape pull they needed to retract the scapulae for proper posture. The kinesiotape was kept on for a period of five days, and then removed for a period of two days before being reapplied the next day. The tape was applied one time each week over a four-week period. There were no allergic reactions 
to the kinesiotape during the study.

Data Analysis

A coded data sheet (Table C6) contained the subject number and was used for baseline and follow-up measurements. To determine the CVA a still photograph using the Hudl app was used to draw a line from the spinous process of the $\mathrm{C} 7$ vertebrae ${ }^{39}$ to the tragus of the ear and a horizontal line that bisected the spinous process of the C7 vertebrae. ${ }^{39}$ The Hudl app was then used to calculate the angle between the two lines and the angle was recorded on the data sheet. To determine the FSA a still photograph using the Hudl app was used to draw a line from the spinous process of the $\mathrm{C} 7$ vertebrae to the acromion process of the shoulder and a horizontal line that bisected the spinous process of the C7 vertebrae..$^{19,29,39}$ The Hudl app was then used to calculate the angle between the two lines and the angle was recorded on the data sheet. To determine the scoring of scapular dyskinesis, the researcher analyzed the recorded video at a later time by examining each scapula while the subject performed weighted shoulder flexion and abduction. The researcher then scored the scapular dyskinesis using a 0-3 scale for each scapula, for a maximum combined score of $6 .^{38}$ A score of 0 was be used if pain was present during the movement, 1 if there was obvious scapular winging on $3 / 5$ trials in either flexion or abduction, 2 if there was subtle scapular winging on $3 / 5$ trials in either flexion or abduction, and 3 if there was normal scapular motion. ${ }^{24,38}$ Pain was determined by the researcher verbally asking the subjects after the testing. The scoring was recorded on the data sheet. The analysis procedure was completed for both the baseline and follow-up measurements.

Statistical Analysis

Descriptive analysis consisted of means and standard deviations for CVA, FHA, and scapular dyskinesis score for each group (exercise and kinesiotape). Three separate $2 \times 2$ 
repeated measures ANOVA were used to determine between group differences (exercise and kinesiotape) for both the pre and posttest measurements; one for the CVA, one for the FHA, and then one for the scapular dyskinesis score. A repeated measures ANOVA was used under the assumptions that there was homogeneity of variance, normal data, and linearity of data. No correction for multiple comparisons was made; instead Cohen d measures of effect size was calculated based on the mean differences of test scores (pretest and posttest) divided by the reference SD (pretest) with corresponding 95\% confidence intervals (CI). The strength of effect

sizes used was small $(\leq .4)$, moderate $(.41-.7)$, and large $(\geq .71) .{ }^{40}$ Three correlations were used to determine the relationship between cell phone and computer use to CVA, FHA, and scapular dyskinesis score. The strength of correlation coefficients used were weak (<.3), moderate (.3-.6), and large (>.6). ${ }^{40}$ The level of significance for all analyses was be $\mathrm{P}=.05$. Minimal detectable change was determined by first calculating the internal reliability, Cronbach's alpha $\left(\mathrm{r}_{\mathrm{x}}\right)$, then using a formula to compute the standard error of the mean $(\mathrm{SEM}),\left(\sqrt{ } 1-\mathrm{r}_{\mathrm{x}}\right)$, and then further the MDC, $(\sqrt{2}) *$ SEM. IBM/SPSS software (IBM/SPSS, Inc., Chicago, IL) version 24.0 was used for all analyses.

\section{RESULTS}

Overall means and standard deviations for all subjects' baseline measurements can be found in Table D1. A two-tailed T-test was run to compare the baseline measurements for the CVA, RSA, and scapular dyskinesis score between groups. There were no significant differences between groups at baseline. (Table D1) A Pearson Product Moment Correlation was run to determine if there was a relationship between the duration of computer and cellphone use and the extent of FHP, RSP, and/or scapular dyskinesis at baseline. There was a negative, weak correlation between cell phone use and CVA $(\mathrm{r}=-.245, \mathrm{p}=.299)$, RSA $(\mathrm{r}=-.253, \mathrm{p}=.281)$, and 
scapular dyskinesis score $(\mathrm{r}=-.208, \mathrm{p}=.378)$. There was a negative, weak correlation between computer use and CVA $(\mathrm{r}=-.147, \mathrm{p}=.538)$ and scapular dyskinesis score $(\mathrm{r}=-.168, \mathrm{p}=.479)$. Computer use and RSA demonstrated a positive, weak correlation. $(r=.007, p=.976)$ All correlations had high p-values and were not significant. There were no time-by-group interaction or differences between groups for the CVA, RSA, and scapular dyskinesis score. There was a main time effect for the scapular dyskinesis score in relation to post-test but not for the CVA and RSA. (Table D2) Effect sizes for both time and group are included in table D3 and D4, respectively. Mean group differences and Minimal detectable change (MDC) for all variables are included in Table D5.

Craniovertebral Angle

There was no significant difference found between time $(\mathrm{F}=2.587, \mathrm{p}=.125)$, group $(\mathrm{F}=.053, \mathrm{p}=.820)$, or time $\mathrm{x}$ group $(\mathrm{F}=1.125, \mathrm{p}=.303)$ for the CVA. Means and standard deviations can be found in Table D2. Effect size calculation was run and determined there was a moderate effect ES=.53 (-.36 to 1.42) for the exercise group pre-test/post-test and a small effect for the kinesiotape group pre-test/post-test ES=.13 (-.75 to 1.00). (Table D3) There was a small effect size for the exercise group in relation to the KT group during post-testing measurements, $\mathrm{ES}=.24$ (-.64 to 1.12). (Table D4) MDC for the CVA measurement was calculated to be .755 . The group mean difference for the CVA was 3.90 and .80 for the exercise and KT group, respectively. The exercise and KT group had a group mean difference for the CVA that was greater than the MDC. (Table D5)

Rounded Shoulder Angle

There was no significant difference found between time $(\mathrm{F}=.148, \mathrm{p}=.705)$, group $(\mathrm{F}=.024$, $\mathrm{p}=.878)$, or time $\mathrm{x}$ group $(\mathrm{F}=593, \mathrm{p}=.451)$ for the RSA. Means and standard deviations can be 
found in Table D2. Effect size calculation was run and determined there was a small effect $\mathrm{ES}=.15$ (-.73 to 1.03) for the exercise group pre-test/post-test and a moderate effect for the kinesiotape group as well ES=-.46 (-1.35 to .43). (Table D3) There was a small effect size for the exercise group in relation to the $\mathrm{KT}$ group during post-testing measurements, ES=.25 (-.63 to 1.13). (Table D4) MDC for the RSA measurement was calculated to be 1.044. The group mean difference for the RSA was .90 and -2.70 for the exercise and KT group, respectively. The KT group had a group mean difference for the CVA that was greater than the MDC but the exercise group did not. (Table D5)

Scapular Dyskinesis Score

There was a significant difference found for time $(\mathrm{F}=12.570, \mathrm{p}<.01)$ but not between time $\mathrm{x}$ group $(\mathrm{F}=.669, \mathrm{p}=.424)$ or group $(\mathrm{F}=1.148, \mathrm{p}=.298)$ for the scapular dyskinesis score. Means and standard deviations can be found in Table D2. Effect size calculation was run and determined there was a moderate effect for both the exercise group, $\mathrm{ES}=.44$ (-.45 to 1.33) and for the kinesiotape group, $\mathrm{ES}=.50$ (-.39 to 1.39) in relation to pre-test/post-test. (Table D3) There was a small effect size for the exercise group in relation to the KT group during post-testing measurements, ES=.36 (-.52 to 1.25). (Table D4) MDC for the scapular dyskinesis score was calculated to be .494 . The group mean difference for the scapular dyskinesis score was .50 and .80 for the exercise and KT group, respectively. Both the exercise and KT group had a group mean difference for the scapular dyskinesis score that was greater than the MDC. (Table D5)

\section{DISCUSSION}

The main purpose of this study was to determine the effects of an exercise versus a kinesiotape intervention on forward head and rounded shoulder posture as well as scapular dyskinesis. The secondary purpose was to determine if there was a correlation between the 
duration of computer and cell phone and the extent of FHRSP and scapular dyskinesis. No significant correlation between duration of computer and cell phone use and the extent of FHRSP and scapular dyskinesis was found. There was no significant group effect or time $\mathrm{x}$ group interaction for the CVA, RSA, and scapular dyskinesis score. There was a time effect for the scapular dyskinesis score by not for the CVA or RSA. For the eight experimental hypotheses, none were accepted, due to the lack of significance. As this is the first study to examine the effects of an exercise versus a kinesiotape intervention on FHRSP and scapular dyskinesis in college-aged students, the ability to compare significant findings is limited.

Cellphone and Computer Use

Technology has advanced in recent years with the addition of smart phones and tablets, and the time spent on those digital devices has increased significantly. ${ }^{10}$ A multivariate regression analysis found that a predictable factor for lower back and neck pain was the amount of cell phone, tablet and computer use. ${ }^{10}$ Increased use of digital technology forces the body to adapt and maintain a flexed, forward posture that is necessary to look at and use the technology. ${ }^{11}$ Holding a posture for an extended duration of time has been the common theme in the general population, industrial setting, and in those who spend prolonged time using digital technology. ${ }^{11,17}$ In this study, the negative correlation found between cell phone use and CVA, RSA, and scapular dyskinesis score, as well as between computer use and CVA and scapular dyskinesis score indicated an inverse relationship between technology use and posture. Meaning that as the use of technology increased, the extent of the FHRSP and scapular dyskinesis worsened. However, the correlation was small and did not warrant any significance. A decrease in CVA and RSA is associated with a worsening posture (i.e. greater extent of forward head and rounded shoulder posture). A decrease in scapular dyskinesis score is associated with a 
worsening of scapular dyskinesis and scapulohumeral rhythm. ${ }^{23-26}$ These correlations are similar to results that have been noted in previous research. ${ }^{10,11,17}$

Studies ${ }^{10-12}$ have shown that individuals who use smartphones and personal technology tend to have a forward and flexed posture (FHRSP) and an increase in neck and shoulder pain. The FHP is due to the upper cervical region being placed into extension and the lower cervical region into flexion, both in the sagittal plane; ${ }^{11}$ the smartphones and tablets force the user to use a flexed neck posture, thus decreasing CVA, RSA and a decrease in scapular dyskinesis score. The decrease in CVA and RSA is associated with a worsening posture (i.e. greater extent of forward head and rounded shoulder posture). A decrease in scapular dyskinesis score is associated with a worsening of scapular dyskinesis and scapulohumeral rhythm. ${ }^{23-26}$ As increases in CVA, RSA and scapular dyskinesis scores were noted in this study, further studies are warranted to note whether increases or decreases occur with an increase in cellphone use and in the type of cellphone use whether it be in terms of calling, texting, and/or gaming, all of which require a different posture for various amounts of time.

Forward Head/Rounded Shoulder Posture

The CVA and RSA are commonly used for objectifying the extent of FHRSP. ${ }^{29,37,39}$ What was not detected statistically in this study in relation to time, group or time, by group interaction, a clinical meaningful difference was noted. There were some changes evident as the exercise group had an average increase for the CVA and RSA measurements in relation to pre-test/posttest, while the kinesiotape group only showed an increase in CVA compared to baseline. Over the four weeks, moderate post-test effect sizes indicated that a change in CVA and RSA started to occur compared to baseline measurements. However, most of the positive effects favored the exercise group and not the kinesiotape group. The kinesiotape group did have a decrease in RSA 
compared to baseline, meaning that the extent of forward shoulder posture actually worsened (decreased) over the duration of the study; this was postulated to be based on the pattern of the kinesiotape in relation to the scapula area. The rounded shoulder angle uses a line from the $7^{\text {th }}$ cervical spinous process to the acromion process and a horizontal line through the acromion. Where the kinesiotape was placed favored the position of the scapula and not the rounded position of the shoulder. There would be no contact with the $\mathrm{C} 7$ vertebrae and acromion. Although research on the use of KT for correcting FHRSP is limited to one case study, the KT intervention was an effective treatment. ${ }^{36}$ Further study of kinesiotape is warranted, especially using a pattern that would favor placing the tape closer to the $\mathrm{C} 7$ vertebrae and the acromion to increase the RSA and improve RSP.

The CVA is a measure of the angle between a horizontal line using the body of the $7^{\text {th }}$ cervical vertebra and a line from the $7^{\text {th }}$ cervical vertebra to the tragus of the ear. ${ }^{14}$ Placement of the kinesiotape in this study appeared to have no influence on CVA as well. The KT protocol used in this study was to correct the FHRSP and scapular dyskinesis, therefore again not having any connection with the cervical vertebrae. The KT was applied over the back and posterior shoulder and not the cervical region at all. The exercise protocol may have a better relationship for the change in CVA because a number of the exercises were directly aimed at correcting a FHP as opposed to the KT intervention that did not directly affect the FHP. As the exercise group benefited more than the kinesiotape group, perhaps a better relationship may be evident if exercise and kinesiotape are used at the same time instead of in isolation as standalone treatments.

Previous research ${ }^{19,23,29-31}$ has shown that an exercise protocol has been effective in treating and correcting FHRSP. The exercise group did display an overall improvement in 
FHRSP, which could be related to the exercises that were incorporated during the four weeks of the study. Two similar exercise protocols were completed throughout this study, one was a HEP and the other was completed with direct assistance. Both sets of exercises were specifically designed to correct FHRSP, by stretching specific muscles groups such as the pectoralis minor and sternocleidomastoid. However, the direct assistance exercises included both stretching and strengthening by using a manual stretch or appropriate manual resistance by following PNF diagonal patterns. Why these exercises were selected in based on the influence of the pectoralis minor on RSP due to the muscular attachment at the coracoid process. Tightness in the pectoralis has been correlated with a worsening RSP and has been measured through the pectoralis minor index. ${ }^{19}$ The index measures the length of both pectoralis minor muscles with a comparison of the acromion to table distance to correlate the tightness of the muscles with RSP. ${ }^{19}$ Stretching the pectoralis minor has exhibited a significant decrease in the extent of RSP and thus the reason the stretch was included in this current study. ${ }^{31}$ The subjects in the exercise group were assisted with exercises once a week. With assistance only once a week over the fourweek period, this may be the reason why the exercise group did not improve any better than the kinesiotape group. In a study ${ }^{23}$ using exercises to improve FHRSP, subjects were assisted with exercises twice a week. In that study there was a significant decrease in the CVA as compared to a control. Based on results from this study that started to indicate a change in a four-week period, additional studies should increase the length of the study and the number of times per week when the assisted exercises are incorporated each week.

Posture has been revealed as a main culprit of shoulder and neck pain through previous research. ${ }^{6,13-16}$ The chosen exercises targeted the muscle imbalances that created the postural distortion. Forward head posture can occur with tight sternocleidomastoid muscles and 
weakened deep neck flexor muscles. ${ }^{14,16}$ Stretching of the sternocleidomastoid was used to decrease the muscle tightness while the chin tuck exercise was used to activate the deep neck flexors. A proper muscular balance between the sternocleidomastoid and the deep neck flexors should theoretically correct forward head posture. Rounded shoulder posture occurs when the pectoralis major and anterior chest muscles become tightened while the posterior thoracic muscles and scapular stabilizers are weakened. ${ }^{14,16}$ A stretch of the pectoralis minor while activating the posterior thoracic muscles and scapular stabilizers through the Y's to I's and scapular punches exercises facilitate the correct muscular balance.

Scapular Dyskinesis

Both the exercise and kinesiotape group displayed an increase in scapular dyskinesis score from baseline. This change was also evident clinically as both groups started to note improvement, with moving towards a higher combined score of 6 , which corresponds with normal bilateral scapular motion. Despite the brief period of the four-week exercise intervention, changes were becoming evident for scapular dyskinesis scores, but the changes were also evident for the kinesiotape group. Improvement of scapular dyskinesis is dependent on correcting the improper scapular mechanics through increasing the neuromuscular control and strength of the scapular stabilizers. ${ }^{23}$ Those two factors were the main focus of the wall wash, Y's to I's, scapular PNF, and scapular punch exercises. The kinesiotape intervention, on the other hand, was intended through previous research and theory, to act as a proprioceptive reminder for the subjects to correct the improper posture and activate the scapular stabilizers. ${ }^{33-36}$ The reason why $\mathrm{KT}$ is considered a proprioceptive intervention is based on stimulating superficial sensory nerves that are reacting to the KT as the stimuli. ${ }^{33}$ However, since both intervention groups achieved MDC for the scapular dyskinesis score, it is evident that changes in baseline measurements were 
present, but a longer intervention may have led to even higher scores. Without current research on the use of the KT for correction of scapular dyskinesis or the length of time to incorporate an exercises protocol to demonstrate meaningful improvement for the individual, only speculation can be used. At this time only best clinical practice dictates what protocol to follow. Therefore it is not known whether a four-week intervention may have been too short of duration to reflect changes in scapular scores from baseline.

Scapular dyskinesis is defined as an alteration in the kinematics of the scapula itself. The altered kinematics distort force-couples and length-tension relationships, therefore a rehabilitation program for scapular dyskinesis must incorporate exercises designed to regain neuromuscular control of scapular stabilization muscles, ${ }^{23}$ or the use of kinesiotape to proprioceptively change the position of the scapula. The rehabilitation and treatment of scapular dyskinesis focuses on stretching the pectoralis minor, while strengthening and regaining control of the scapular stabilizers using closed kinetic chain exercises. ${ }^{23}$ Strengthening exercises for scapular stabilizers involve scapular depression, elevation, protraction, and retraction, as well as Y's to I's. ${ }^{23,29}$ The Y's to I's exercise involves the patient retracting both scapulae while the shoulders are abducted 90 degrees and elbows flexed 90 degrees. The elbows are then brought into extension while the shoulder continues into further abduction above the head while keeping the scapula retracted. ${ }^{29}$ The most important concept with scapular dyskinesis rehabilitation is adherence to a maintenance exercise program focused on scapular stabilization and neuromuscular control. It has been shown the overhead athletes with pain during throwing all returned to pain-free throwing within 3-4 months of undergoing a scapular strengthening program and all but nine athletes remained pain-free after 1 year. Those nine athletes admitted to non-adherence to the maintenance program. ${ }^{23}$ 
Several techniques have been used to evaluate scapular dyskinesis. Most require expensive equipment that is not available to the clinician ${ }^{26}$ or rely on subjective judgment whether scapular dyskinesis is obvious, subtle or normal ${ }^{24-25}$ while performing static or dynamic movement. $\mathrm{McClure}^{24}$ determined there was a $75-82 \%$ agreement between raters when scapular dyskinesis is classified as normal, subtle, or present. Butowicz ${ }^{38}$ adapted the classification system by giving a numerical value for scapular motion. Pain during movement was scored as a 0 , obvious scapular winging during movement was scored as a 1 , subtle winging scored a 2 , and normal scapular motion scored a 3 . This was completed for both scapulae for a maximum combined score of $6 .^{38}$ This scoring method used was developed to objectively measure scapular dyskinesis by putting a score to the extent of scapular dyskinesis present. However, it should be noted that there are no current reliability or validity studies for the scapular dyskinesis scoring method. Therefore, further clinical studies are warranted to find a technique that will help the clinician determine whether the treatment techniques used in rehabilitation are effective or not. The use of the Hudl app was effective in determining CVA and RSA; perhaps this could also be incorporated to determine scapula dyskinesis.

\section{Clinical Importance}

Although this study found statistical significance only over time for the scapular dyskinesis score, the benefits lie within the clinical relevance. The results of the correlations in this study indicate that overall there was a weak, yet negative relationship between computer/cellphone use and extent of FHRSP as well as scapular dyskinesis. The weak correlation in this study deems that the results are inconclusive on whether the use of technology had an influence over FHRSP and scapular dyskinesis. This is opposite to what other studies have found. Previous research has shown that there is a strong relationship between technology 
use and poor posture and improper scapular mechanics. ${ }^{10-12}$ Poor posture and improper scapular mechanics have also been shown to be a major factor in neck, shoulder, and overall back pain. ${ }^{6,13-16}$ Despite the sample used in this study consisting of healthy, active, college age students, the negative correlation was still present and should be taken into consideration when attempting to treat neck, shoulder, and back pain by correcting posture and scapular mechanics. The use of technology was not controlled throughout this study and it very well may have played a vital role in the lack of significance. On the other, based on the subject population in this study, time spent on cell phones throughout the day may be minimal. During the day the subjects were in classes in the morning and in the athletic training rooms in the afternoon, where use of cell phones is discouraged.

Previous research has shown that therapeutic exercises are successful in treating FHRSP as well as scapular dyskinesis. ${ }^{19,23,29-32}$ For this study the exercise protocol was based on previous research that was successful at improving FHRSP and scapular dyskinesis. The exercise protocol incorporated in this study was aimed at correcting the muscular imbalances through stretching and strengthening. Overall, therapeutic exercises have shown success in treating FHPRSP and scapular dyskinesis but which exercises to use still remains best clinical practice. ${ }^{19,23,29-32}$ In addition, the KT protocol has not been examined outside of this current study in relation to treating FHRSP and scapular dyskinesis and thus the use is considered best clinical practice based perhaps on previous research ${ }^{33-35}$ or lack of research.

Many of the subjects involved in this study were at the high end of measurements during baseline testing, meaning that the FHRSP and scapular dyskinesis was not that severe. This could attribute to the lack of significance found in this study. Most of the subjects improved in 
terms of FHRSP and scapular dyskinesis, however since they did not exhibit extreme postural distortions that may be a leading factor for the lack of significant improvement.

Limitations of the Study

Several limitations have been identified in this study. The first is the small sample size $(n=20)$ used, which was due to use of a convenience sample. A convenience sample of healthy, non-athletic, college age students was used. Therefore, the results cannot be generalizable to all college students. The length of the intervention of the study (4 weeks) as well as only one meeting per week was also identified as a limitation. However, there are no studies in the literature indicating as to what the length should be. No compliance log was kept and should be noted as a limitation as well, as the subjects only verbally stated they were completing the exercises. The self-assessed cellphone use was not specific in terms of calling, texting, and/or gaming on the cellphone, all of which require a different posture for various amounts of time. There was no blinding of the assessor and the therapist instituting the interventions. In addition, use of the scapular scoring method is relatively new with few reliability and validity studies. It is quite possible that there was measurement error on the researchers part as well as a practice effect from pre to post testing, but SEMs were fairly low. The use of the Hudl app may also be a limitation, as the validity and reliability of the Hudl app for measuring the CVA and RSA has not been determined. Thus, further studies are warranted on the use of the scapular dyskinesis scoring method and the Hudl app.

\section{CONCLUSION}

The results from this study indicate that the only significant finding was for the scapular dyskinesis score where post-test measurements improved for both groups and both groups achieved MDC. However, moderate effects indicated clinically that change was starting to 
occur. In addition, there was a weak, negative, but non-significant relationship between computer/cellphone use and FHRSP and scapular dyskinesis. Thus, additional research should be conducted by using a larger population to verify or refute the results of this study. As only healthy subjects were used, use of an athletic or injured population should be considered. 


\section{REFERENCES}

1. Hoy, DG, Protani M, De R, Buchbinder R. The epidemiology of neck pain. Best Pract Res Cl Rh. 2010;24(6):783-792.

2. Hogg-Johnson, S, van der Velde G, Carroll LJ, Holm LW, Cassidy JD, Guzman J, Cote P, Haldeman, Ammendolia C, Carragee E, Hurwitz E, Nordin M, Peloso P. The burden and determinants of neck pain in the general population. Eur Spine J. 2008;17(1):39-51.

3. Feleus A, Bierma-Zeimstra SM, Bernsen RM, Miedema HS, Verhaar JA, Koes BW. Management decisions in nontraumatic complaints of arm, neck, and shoulder in general practice. Ann Fam Med. 2009;7(5):446-454.

4. Luime, JJ, Koes BW, Hendriksen IJ, Burdorf A, Verhagen AP, Miedema HS, Verhaar JA. Prevalence and incidence of shoulder pain in the general population; a systematic review. Scand J Rheumatol. 2004;33(2):73-81.

5. McBeth J, Jones K. Epidemiology of chronic musculoskeletal pain. Best Pract Res Cl Rh. 2007;21(3):403-425.

6. Siivola, SM, Levoska S, Latvala K, Hoskio E, Vanharanta H, Keinänen-Kiukaanniemi S. Predictive factors for neck and shoulder pain: a longitudinal study in young adults. Spine. 2004;29(15):1662-1669.

7. Straker LM, O'Sullivan PB, Smith AJ, Perry MC. Relationships between prolonged neck/shoulder pain and sitting spinal posture in male and female adolescents. Man Ther. 2009;14:321-329.

8. Hanvold TN, Veiersted KB, Waersted M. A prospective study of neck, shoulder, and upper back pain among technical school students entering working life. $J$ Adolescent Health. 2010;46:488-494.

9. Reeser JC, Joy EA, Porucznik CA, Berg RL, Colliver EB, Willick SE. Risk factors for volleyball-related shoulder pain and dysfunction. Am Acad Phy Med Rehabil. 2010;2:2736.

10. Shan Z, Deng G, Li J, Li Y, Zhang Y, Zhao Q. Correlational analysis of neck/shoulder pain and lower back pain with use of digital products, physical activity and psychological status among adolescents in Shanghai. Plos Med. 2013;8(10):1-9.

11. Kim MS. Influence of neck pain on cervical movement in the sagittal plane during smartphone use. J Phys Ther Sci. 2015;27(1):15-17.

12. Shabbir M, Rashid S, Umar B, Ahmad A, Ehsan S. Frequency of neck and shoulder pain and use of adjustable computer workstation among bankers. PakJ Med Sci. 2016;32(2):423-426. 
13. Harrison DD, Harrison DE, Janik TJ, Cailliet R, Ferrantelli JR, Haas JW, Holland B. Modeling of the sagittal cervical spine as a method to discriminate hypolordosis: results of elliptical and circular modeling in 72 asymptomatic subjects, 52 acute neck pain subjects, and 70 chronic neck pain subjects. Spine 2004;29(22):2485-2492.

14. Ting Yip CH, Wing Chiu TT, Kuen Poon AT. The relationship between head posture and severity and disability of patients with neck pain. Man Ther. 2008;13:148-154.

15. Lluch E, Arguisuelas MD, Quesada OC, Noguera EM, Puchades MP, Rodriguez JA, Falla D. Immediate effects of active versus passive scapular correction on pain and pressure pain threshold in patients with chronic neck pain. J Manip Physiol Ther. 2014;37(9):660-666.

16. Silva, AG, Johnson ML. Head posture and neck pain of chronic nontraumatic origin: a comparison between patients and pain-free persons. Arch Phys Med Rehabil. 2009;90(4):669-674.

17. Aaras A, Fostervold KI, Ro O, Thoresen M. Postural load during VDU work:a comparison between various work postures. Ergonomics. 1997;40:1255-68.

18. Braun BL. Postural differences between asymptomatic men and women and craniofacial pain patients. Arch Phys Med Rehabil. 1991;72:653-56.

19. Lee J, Cynn H, Yi CH, Kwon O, Yoon TL. The effect of scapular posterior tilt exercise, pectoralis minor stretching, and shoulder brace on scapular alignment and muscles activity in subjects with round-shoulder posture. J Electromyogr Kines. 2015;25(1):107114.

20. Ludewig PM, Cook TM. Alteration in shoulder kinematics and associated muscle activity in people with symptoms of shoulder impingement. Phys Ther. 2000;80(3):276-291.

21. Weon, JH, Oh JS, Cynn HS, Kim YW, Kwon OY, Yi CH. Influence of forward head posture on scapular upward rotators during isometric shoulder flexion. J Bod Movement Ther. 2010;14(4):367-374.

22. Cools AM, Struyf F, De Mey K, Maenhout A, Castelein B, Cagnie B. Rehabilitation of scapular dyskinesis: from the office worker to the elite overhead athlete. Br J Sports Med. 2014;48:692-697.

23. Burkhart SS, Morgan CD, Kibler WB. The disabled throwing shoulder: spectrum of pathology part III: the SICK scapula, scapular dyskinesis, the kinetic chain, and rehabilitation. Arthrosc. 2003;9(7):641-661.

24. McClure P, Tate AR, Kareha S, Irwin D, Zlupko E. Clinical method for identifying scapular dyskinesis, part 1: reliability. J Athl Train. 2009;44(2):160-164. 
25. Kibler BW, Uhl TL, Maddux JWQ, Brooks PV, Zeller, B, McMullen J. Qualitative clinical evaluation of scapular dysfunction: a reliability study. J Shoulder Elbow Surg. 2002;11(6):550-556.

26. Uhl TL, Kibler BW, Gecewich B, Tripp BL. Evaluation of clinical assessment methods of scapular dyskinesis. Arthroscopy. 2009;25(11):1240-1248.

27. Huang TS, Huang HY, Wang TG, Tsai YS, Lin JJ. Comprehensive classification test of scapular dyskinesis: a reliability study. Man Ther. 2015;20:427-432.

28. Wang C, Mcclure P, Pratt NE, Nobilini R. Stretching and strengthening exercises: their effect on three-dimensional scapular kinematics. Arch Phys Med Rehabil 1999;80(8):923929.

29. Ruivo, R.M., A.I. Carita, and P. Pezarat-Correia. The effects of training and detraining after an 8 month resistance and stretching training program on forward head and protracted shoulder postures in adolescents: randomised controlled study. Man Ther. 2016;21:76-82.

30. Gupta BD, Aggarwal S, Gupta B, Gupta M, Gupta N. Effect of deep cervical flexor training vs. conventional isometric training on forward head posture, pain, neck disability index in dentists suffering for chronic neck pain. J Clin Diag Res. 2013;7(10):2261-2264.

31. Wong CK, Coleman D, diPersia V, Song J, Wright D. The effects of manual treatment on rounded-shoulder posture, and associated muscle strength. J Bod Mov Ther. 2010;14:326333.

32. Struyf F, Nijs J, Mottram S, Roussel NA, Cools AMJ, Meeusen R. Clinical assessment of the scapula: a review of the literature. Br J Sport Med. 2012:1-8.

33. Miralles I, Monterde S, Rio O, Valero S, Montull S, Salvat I. Has Kinesio tape effects on ankle proprioception? A randomized clinical trial. Clin Kinesiol. 2014;68(2):9-18.

34. Gramatikova M, Nikolova E, Mitova S. Nature, application, and effect of Kinesio-taping. Act Phys Edu Sport. 2014;4(2):115-119

35. Nelson NL. Kinesio taping for chronic low back pain: a systematic review. J Bod Mov Ther. 2016:1-10.

36. Hwang-Bo G, Lee JH, Kim HD. Efficacy of kinesiology taping for recovery of dominant upper back pain in female sedentary worker having a rounded shoulder posture. Technol Health. 2013;21:607-612. 
37. Cheung-Lau HM, Wing Chiu TT, Lam TH. Clinical measurement of craniovertebral angle by electronic head posture instrument: a test of reliability and validity. Man Ther. 2009;14:363-368.

38. Butowicz C. (2016). Comprehensive performance-based movement system screening tool for athletes. [dissertation]. Philadelphia: Drexel University; 2016

39. Thigpen, CA, Padua DA, Michener LA, Guskiewicz K, Giuliani C, Keener JD, Stergiou N. Head and shoulder posture affect scapular mechanics and muscle activity in overhead tasks. J Electromyogr Kines. 2010;20(4):701-709.

40. Cohen J. Statistical Power Analysis for the Behavioral Sciences. 2nd ed. Hillsdale, NJ: Lawrence Erlbaum; 1988. 


\section{APPENDICES}




\section{APPENDIX A}

\section{THE PROBLEM}

\section{Research Question}

Neck pain in the general population has ranged from $.4 \%$ to $86.8 \%,{ }^{1} 30 \%-50 \%,{ }^{2}$ or $31 \%,{ }^{3}$ while also having an incidence rate of $10.4 \%$ to $21.3 \%$ in one year. ${ }^{1}$ The cause of neck pain in the study included, but was not limited to disc protrusion/herniation, neck sprain/strain, and/or other soft tissue injuries. ${ }^{2}$ However, despite the prevalence of certain injuries, research has also shown that patients suffering from upper cervical and glenohumeral joint (GHJ) pain do not always have an underlying pathology. ${ }^{14}$ Patients with neck pain have been found to have a smaller angle in relation to the tragus of the ear and the $\mathrm{C} 7$ vertebra, measured from the horizontal; this measurement is otherwise known as the craniovertebral $(\mathrm{CV})$ angle. ${ }^{14}$ The smaller the CV angle the more pronounced forward head posture (FHP) the patient will have, and will depict an inverse relationship with the amount of neck pain (smaller angle, greater amount of neck pain). ${ }^{14}$ Younger patients ( $<50$ years old) have been found to have FHP more frequently and thus have a statistically significant increase in neck pain. ${ }^{16}$ The reasoning behind FHP posture causing neck pain is that upper cervical extension and anterior translation of the vertebrae is believed to place a compressive force on the vertebra while the FHP also lengthens the neck flexors and shortens the neck extensor muscles. ${ }^{16}$ Neck flexors include the sternocleidomastoid (SCM), while the upper trapezius (UT) is classified as a posterior neck extensor muscle.

Another non-traumatic yet, chronic condition in the general population is GHJ pain. 6.7$66.7 \%,{ }^{4}$ or $10 \%-57.9 \%{ }^{5}$ of the general population will have GHJ pain at some point in their lifetime. ${ }^{4}$ Further, research has shown an increase in prevalence in both neck and GHJ pain in 
adolescents from $17 \%$ to $28 \% .{ }^{6}$ Of patients with glenohumeral joint pain, $16 \%-40 \%$ have an associated shoulder impingement as the root cause of the pain. ${ }^{20}$ Shoulder impingement, though highly variable, can be summarized as a decrease in subacromial space preventing adequate clearance of the supraspinatus tendon. ${ }^{20}$ Kinematic analysis of patients with shoulder impingement reveal excessive anterior/posterior translation of the humeral head, inadequate external rotation of the humerus, and a decrease in the normal upward rotation of the scapula. ${ }^{20}$ The above mentioned abnormal shoulder kinematics are associated with a rounded shoulder posture, which may cause a decrease in activity for the lower trapezius (LT) and serratus anterior (SA) muscles as well as hyperactivity of the pectoralis minor (PM). ${ }^{19}$ This imbalance causes a disturbance of the force couple that acts on the scapula to create ideal rotation. ${ }^{20}$ Improper rotation of the scapula is better known as scapular dyskinesis and is a major predictor of pathology in patients with shoulder impingement. ${ }^{39} \mathrm{FHP}$ also has an influence on the scapular mechanics in that a FHP decreases the activity of the SA and LT while increasing the muscle activation of the UT during shoulder flexion, further distorting scapulohumeral rhythm and is know as scapular dyskinesis. ${ }^{21}$

A specific type of scapular dyskinesis is SICK scapula, which the acronym stands for scapular malposition, inferior medial border prominence, coracoid pain and malposition, and dyskinesia of scapular movement. ${ }^{23-26}$ Abnormal posture, as with FHRSP, causes the scapula and glenohumeral joint to be abnormally positioned, which changes the relationship of forcecouples as well as length-tension relationships. ${ }^{16-23}$

FHRSP has been observed and researched in the general population and more recently in the industrial setting as well. ${ }^{17}$ Studies conducted within the industrial setting have shown that individuals that sit at a computer, desk, or hold a certain posture for an extended duration of time 
tend to experience neck and back pain. ${ }^{17}$ Further analysis examined that a FHRSP is the most common dysfunction that causes the pain. ${ }^{18}$ The prolonged posture will begin to tighten the PM, SCM, UT, and levator scapulae, while stretching and causing fatigue in the LT, and SA. ${ }^{16,19}$ Thus, postural correction is a necessary and successful treatment for FHRSP in the industrial/work-place setting. ${ }^{41}$ It has also been found that a sensory feedback system, both visual and audio, was successful in decreasing the FHRSP as well as neck and back pain, while also preventing work-place injuries. ${ }^{41}$

In addition to industrial settings, FHRSP has been noted in the younger population due to technological innovations. Technology has advanced in recent years with the addition of smart phones and tablets, and the time spent on those digital devices has increased significantly. ${ }^{10} \mathrm{~A}$ multivariate regression analysis found that a predictable factor for lower back and neck pain was the amount of cell phone, tablet and computer use. ${ }^{10}$ Increased use of digital technology forces the body to adapt and maintain a flexed, forward posture that is necessary to look at and use the technology. ${ }^{11}$ Holding a posture for an extended duration of time has been the common theme in the general population, industrial setting, and in those who spend prolonged time using digital technology. ${ }^{11,17}$ That same posture can be prevalent and carry over into athletics when an activity or motion is repeated multiple times during a practice, game, or training session. ${ }^{9}$

Posture has been linked to chronic back, neck, and GHJ pain as well as a variety of injuries. ${ }^{14}$ Poor posture in athletics may lead to an increased risk of injury in addition to a decrease in athletic performance. ${ }^{9}$ Although there is little known about the direct correlation between posture, injuries, and performance, restoration of correct posture has become a focal point of rehabilitation in the athletic and general populations. ${ }^{9,23}$ 
Treatment for shoulder and neck pain caused by forward head/rounded shoulder posture has been the focus for physical therapists, athletic trainers, and physicians alike. The treatment for rounded shoulders includes stretching of the PM, SCM, UT, and levator scapulae muscles while strengthening the LT, SA, rhomboids, and the rotator cuff muscle group. ${ }^{19,29}$ Treatment for forward head posture has included stretching of the SCM, while strengthening the deep cervical flexors. ${ }^{42}$ The treatment for FHRSP has typically relied on only manual therapy as an effective way of managing the postural deviation. ${ }^{19,29,42} \mathrm{~A}$ tightening of the posterior neck muscles and anterior thoracic muscles combined with an inhibition of the anterior cervical muscles and posterior thoracic muscles causes FHRSP. ${ }^{16,19}$ The treatment for Scapular dyskinesis is similar to that of FHP and RSP due to the relationship of the pathologies. ${ }^{27,32}$ Scapular dyskinesis rehabilitation protocols should include strengthening of the LT, SA, deep cervical flexors, and the scapular stabilizers while stretching the posterior glenohumeral joint capsule, UT, and PM. 23,27,32 Usually strengthening and stretching exercises for the glenohumeral joint, neck, and scapular stabilizers include chin tucks, Y's to I's, wall washes, upright scapula punches, corner stretch, and a static sternocleidomastoid stretch. $23,29-31,43$

Most postural correction rehabilitation focuses on manual therapy of stretching and strengthening muscles. The imbalance of muscles causes a distortion in the force couples acting upon the neck, scapula, shoulder, and spine. ${ }^{20}$ Recently, Kinesiotape (KT), without the use of manual therapy, has been shown to be an effective method for rounded shoulder posture. ${ }^{36}$ However, this was a case report ${ }^{36}$ with only one patient and no control group and the results cannot be rationalized for the general population.

An individual's posture is important not only during athletic and recreational activities but with activities of daily living as well. ${ }^{16}$ The time spent strengthening inhibited muscles and 
stretching overactive muscles has a limit in effectiveness for postural correction. The affected individual must consistently maintain correct posture for differences to occur. ${ }^{23}$ The theory behind $\mathrm{KT}$ is that the KT itself will act as proprioceptive monitor in regard to postural correction. In comparison to a manual therapy rehabilitation treatment session, which is time intensive, a KT intervention could be used in conjunction or act as a standalone treatment. ${ }^{43}$ FHRSP has been shown to be a major factor for increasing pain of individuals with the condition, yet the effects of using KT as a successful intervention for postural correction remains unknown.

As seen with excessive computer terminal use, a prolonged body position can distort posture and cause neck and shoulder pain. ${ }^{17}$ In athletics, numerous sports involve repetitive activities that cause the body to be placed in certain positions multiple times. These sports include, but are not limited to, volleyball, baseball, softball, and swimming. A cross sectional examination of volleyball players found that $60 \%$ reported a history of shoulder problems and that those athletes reporting shoulder pain were more likely to exhibit scapular dyskinesis. ${ }^{9}$ Scapular dyskinesis is associated with a scapula that is tilted anterior, inferiorly, and protracted causing anterior musculature to tighten and posterior musculature to be stretched. ${ }^{23}$ Further, strengthening of the scapular stabilizer muscles and strengthening of the anterior muscles has had success in postural restoration and has shown to decrease pain. However, there is little research on the evaluation and treatment of FHRSP and scapular dyskinesis in the younger and athletic populations. $^{23}$

The shoulder and cervical complex are interconnected through the kinetic chain and force couples, thus FHP and RSP are correlated to a certain degree. ${ }^{21}$ There is an abundance of knowledge on manual treatment and sensory feedback for FHRSP being relatively effective in restoring proper posture and decreasing associated pain in adults and in the industrial setting. 
However, there is lack of knowledge for KT treatment, as well as cervical/GHJ-strengthening and stretching programs for the young, athletic population. Thus, the following questions were asked:

Research Questions

1. Can strengthening of the scapular stabilizer muscles and stretching of the hyperactive muscles affect forward head/rounded shoulders posture as well as scapular dyskinesis over time?

2. Can strengthening of the deep neck flexor muscles and stretching of the posterior neck muscles affect forward head/ rounded shoulders posture as well as scapular dyskinesis over time

3. Can a kinesiotape intervention affect posture; specifically forward head/rounded shoulders posture and scapular dyskinesis over time?

4. Is there a difference between a strengthening and stretching protocol and a kinesiotape intervention with regards to correcting a forward head/rounded shoulders posture?

5. Is there a relationship between the amount of cell phone and computer use and FHP, RSP, and scapular dyskinesis?

Experimental Hypothesis

1. The exercise group will have a greater decrease in rounded shoulder posture compared to the KT group from baseline following a four-week intervention.

2. The exercise group will have a greater decrease in forward head posture compared to the KT group from baseline following a four-week intervention.

3. The exercise group will have a greater decrease in scapular dyskinesis compared to the KT group from baseline following a four-week intervention.

4. A KT intervention will decrease rounded shoulder posture, forward head posture, and scapular dyskinesis from baseline following a four-week intervention.

5. A cervical/GHJ exercise intervention will decrease the rounded shoulder posture, forward head posture, and scapular dyskinesis from baseline following a four-week intervention.

6. There will be a negative correlation between the amount of cellphone and computer use and the CVA. 
7. There will be a negative correlation between the amount of cellphone and computer use and the FSA.

8. There will be a negative correlation between the amount of cellphone and computer use and the score of scapular dyskinesis.

Assumptions

1. All subjects will meet the inclusion criteria for the research study.

2. The instruments used will be valid and reliable.

3. The documentation of each subjects' posture measurement will be accurate.

4. The subjects' posture will be measured before and after a 4-week intervention with identical procedures.

5. The subjects will adhere to the at home exercise protocol.

6. The subjects will adhere to correct techniques for exercises during meetings and at home exercise protocol.

7. The subjects will keep kinesiotape on as instructed if they are in the KT group.

8. The subjects will inform the PI and/or or Co-PI for any allergic reaction to the KT.

Delimitations

1. Subject population is not generalizable to the athletic population. Subject population is specific to healthy college students.

2. The participants' are college-aged students at one institution. The subject population is specific only to one institution.

Operational Definitions

1. CV-The craniovertebral angle; "measured between a horizontal line through the spinous process of $\mathrm{C} 7$ and a line from spinous process of $\mathrm{C} 7$ through the tragus of the ear"14

2. FHP - Forward head posture; the lower cervical spine is in flexion and the upper cervical spine is in extension. ${ }^{4}$

3. FHRSP-Forward head rounded shoulder posture; a postural alteration with both a forward head and rounded shoulders. ${ }^{28,44}$ 
4. KT-Kinesiotape; the original elastic adhesive tape that can be used to re-educate the neuromuscular system. ${ }^{34}$

5. Postural Restoration-The process of returning a dysfunctional posture back to normal through a strengthening and stretching program.

6. RSP-Rounded shoulder posture; "forward displacement of the acromion with reference to the $7^{\text {th }}$ cervical spinous process",28

7. Scapular Stabilizer Muscles-A set of muscles that stabilize the scapula due to force couples. The muscles included are the: supraspinatus, infraspinatus, teres minor, subscapularis, teres major, levator scapulae, serratus anterior, and the latissimus dorsi. ${ }^{23}$

8. SICK scapula-An acronym that stands for: scapular malposition, inferior medial border prominence, coracoid pain and prominence, and dyskinesis of scapular movement. ${ }^{23}$

9. Visual Display Terminals — a computer placed upon a desk with a work seat in front of the desk. ${ }^{17,41}$

\section{Limitations}

1. Participants can drop out at any time.

2. The study may not being generalizable to the athletic population.

3. External validity will exist due to the subject population and the choice of subjects.

4. Participants may not adhere to the at home exercise program $100 \%$.

5. The KT may fall off during the duration of the study.

6. The internal factors of the participants cannot be controlled: health, nutrition, and weight training or other exercises programs.

7. The participants may not come back for additional exercises or measurements.

\section{Significance of Study}

There has not been an association of a KT intervention nor a strengthening and stretching program as means to correct FHRSP specifically in the young, athletic population. The majority of literature on correction of posture is limited to work place and industrial studies in clients that use visual display terminals. There has been an increase in smartphone use and in young 
individuals that participate in repetitive overhead sports. The increase forces the body to be in forward posture, both in the cervical region and GHJ, leading to a resting forward posture. The forward posture can include FHRSP and also lead to scapular dyskinesis and other potential painful pathologies. In addition, sports with repetitive motions, as with cell phone use, cause the body to be placed in a forward posture numerous times. This may cause underlying pathologies in the younger, active population in regards to FHRSP and scapular dyskinesis. Results from this study may show a connection with cell phone and computer use to FHRSP and scapular dyskinesis. Rehabilitation of scapular dyskinesis has been successful, only when adherence to the program is maintained. This study can provide a means to determine whether a strengthening and stretching exercise program or a KT intervention is more successful in treating FHRSP, especially in a young, active population.

At the completion of this study, dissemination of information will occur. This information will have a direct affect not only to athletes but also to the general population and all individuals suffering from chronic pain and lingering injuries caused by postural deviations. It will also create more awareness of postural deviations in the younger, athletic population as well as those who use digital technology for extended periods of time. There is limited, but increasing research on the use and effectiveness of KT and an insight would be useful for long-term effects of rehabilitation programs for postural correction and activity modification in athletes and the younger population. This research will be presented at workshops and seminars at local universities. 


\section{APPENDIX B}

\section{LITERATURE REVIEW}

Introduction

Neck and shoulder pain is a common pathology within the general and athletic populations. ${ }^{1,2,45} 30-50 \%$ of the adult population and $21-42 \%$ of the adolescent population can suffer from neck pain, as well as $6.7 \%$ to $66.7 \%$ of the general population will experience shoulder pain within their lifetime. ${ }^{2,4}$ Both of these pathologies can be debilitating and can affect activities of daily living as well as athletic performance. ${ }^{2,4}$ From 1989-1996, the prevalence of neck and shoulder pain in the young adult population increased from 17 to $28 \%$ percent. ${ }^{6}$ This increase was associated with leisure time activities that were not physically engaging. These activities included resting, reading, and/or listening to music. ${ }^{6}$ More recently, studies have shown that there is a relationship in technology use and shoulder and neck pain. Computers, tablets, and smartphones have become part of the general population's every day routine and for

some, a vital part of jobs. ${ }^{10-12}$ One study ${ }^{11}$ has shown that during cellphone/smartphone use there is an increase in cervical flexion, which further correlates to neck pain. The increase in cervical flexion is a postural adaption that causes the head to move forward in the sagittal plane. Similar findings are present with computer and visual display terminals in that individuals with increased time spent at computers are shown to have a forward head and rounded shoulder posture. ${ }^{41}$ Overall the literature on cervical and shoulder posture and the correlation to neck and shoulder pain has increased with the progression in technology, however treatment concerns have not increased at the same rate. In this literature review the following topics will be discussed: forward head and rounded shoulder posture, scapular dyskinesis, the measurement and treatment 
techniques for forward head/rounded shoulder posture and scapular dyskinesis, and kinesiotape uses and application.

Forward Head and Rounded Shoulder Posture

Forward head posture is a deviation of the cervical spine in which the lower cervical spine is held in flexion while the upper is in extension, causing the head to protrude forward in the sagittal plane. ${ }^{44}$ A relationship has been found in individuals who have an increased FHP and cervical spine pain and disability. ${ }^{14}$ The researchers used a head posture spinal curvature instrument to measure the craniovertebral (CV) angle for FHP while the subjects filled out a neck pain questionnaire and a pain rating scale. The subjects with neck pain had a significantly smaller CV angle, meaning a more pronounced forward head. ${ }^{14}$ FHP places an increase pressure on the cervical intervertebral discs and creates a muscular imbalance between the cervical flexors and extensors. ${ }^{16}$ However, the study by Ting Yip et al. ${ }^{14}$ showed that the correlation between FHP and scores on the neck pain questionnaire and the pain rating scale were moderate. These results indicate that FHP is one factor that may cause neck pain. ${ }^{14}$ Silva and Johnson $^{16}$ investigated the relationship between FHP and postural sway, while hypothesizing that FHP and postural would have a negative correlation. After measuring the subjects FHP by means of the $\mathrm{CV}$ angle, the subjects were placed on a force platform to measure postural sway. The researchers found there was no statistically significant relationship between the two factors. ${ }^{16}$ A similar study by Lee ${ }^{19}$ assessed the effects of FHP on both static and dynamic balance control. There was no significant difference in dynamic balance for those subjects with and without FHP, however there was a statistically significant difference in static balance ${ }^{19}$ The results from these studies ${ }^{14,16,19}$ indicate that although FHP has an influence on pain ${ }^{14}$ it may or may not have an influence on balance and further activities of daily living. ${ }^{16,19}$ 
RSP is the forward displacement of the acromion in reference to the $7^{\text {th }}$ cervical spinous process and thus the entire glenohumeral joint structure is affected. ${ }^{28}$ RSP decreases the subacromial space in the glenohumeral joint due to the anterior displacement of the scapula. ${ }^{20}$ Lee et al. ${ }^{46}$ used a regression analysis to determine predictor variables for RSP. The variables examined the pectoralis minor (PM) index, serratus anterior (SA) strength, and posterior shoulder tightness. The researchers measured the length of the PM for the PM index, used a dynamometer to measure the SA strength, and measured glenohumeral horizontal adduction and internal rotation to determine the amount of posterior shoulder tightness. The regression analysis determined that the PM index and posterior shoulder tightness attributed to $83 \%$ of variance for RSP and those factors should be a focus when managing RSP. ${ }^{46}$ In addition, Thigpen et a ${ }^{39}$ compared the muscle activity in individuals with and without FHRSP during over head task. After using surface electromyography, the researchers determined that there was a significant decrease in SA activity but not upper and lower trapezius activity for those subjects with FHRSP compared to those without. These results show that a weakened or inhibited SA is a possible factor in causing FHRSP. ${ }^{39}$

Recently, the literature determined that prolonged posture, as with the use of smartphones, ${ }^{10}$ tablets, ${ }^{10}$ and computers ${ }^{10,41}$ as being a major factor in causing neck and shoulder pain. ${ }^{11,17}$ Additionally, the industrial work setting has shown recent research verifying the use of visual display terminals as a cause. ${ }^{41}$ Lifestyle questionnaires were given to high school students that focused on frequency of neck, shoulder, and back pain, use of digital products, extracurricular activities, and then additional academic pressure and mental status. $40.8 \%$ and $33.1 \%$ of respondents stated they suffered from neck and shoulder pain and lower back pain, respectively. ${ }^{10}$ Those respondents that used mobile phones for greater than two hours a day or 
used a tablet or personal computer at all had a significant increase in prevalence of neck and shoulder pain than those who did not. ${ }^{10}$ However, those participants who reported physical activity 1-4 times weekly also had a decrease in prevalence of neck and shoulder pain. ${ }^{10}$ Further, studies ${ }^{46-50}$ have also shown that individuals who use smartphones and personal technology tend to have a forward posture (FHRSP) and neck pain. The FHP is due to the upper cervical region being placed into extension and the lower cervical region into flexion, both in the sagittal plane; ${ }^{11}$ the smartphones and tablets force the user to use a flexed neck posture. ${ }^{46}$ Scapular Dyskinesis

Scapular dyskinesis has been referred to as the SICK scapula. ${ }^{23}$ The acronym of SICK stands for scapular malposition, inferior medial border prominence, coracoid pain and malposition, and dyskinesia of scapular movement. ${ }^{23-26}$ While SICK scapula is a static measurement, it affects the dynamic movement of the scapula and produces scapular dyskinesia. ${ }^{23}$ A SICK scapular pattern induces pain not only at the coracoid process but in the entire glenohumeral joint due to altered joint positioning, which modifies length-tension and force couple relationships negatively. ${ }^{23}$ SICK scapula is a specific type of scapular dyskinesis, where the kinematics of the scapula is altered. Depending on the source, there are three to four classifications of scapular dyskinesis. Type I is the inferior medial border prominence, type II is a medial scapular border prominence, type III is superiormedial scapular border prominence, and type IV is normal scapular movement. ${ }^{23-27} \mathrm{Uhl}^{26}$ also examined a two-type classification based on whether scapular dyskinesis was present (yes) or absent (no).

Scapular dyskinesis and SICK scapula arises from previous injuries, ${ }^{51,52}$ muscular imbalances, ${ }^{23}$ and/or posture. ${ }^{52}$ Previous injuries to the glenohumeral joint including acromial clavicular separations, impingement, and rotator cuff injuries may leave an individual more 
susceptible to developing scapular dyskinesis. ${ }^{51}$ Muscular imbalances and posture are highly related as discussed with FHRSP and have an influence on the development of scapular dyskinesis. ${ }^{23,52}$ The muscular imbalances occur due to repetitive flexion activities as well as prolonged flexion posture. ${ }^{9,23}$ As with FHRSP, the prolonged flexion posture ensues situations such as extended use of personal technology in terms of smartphones, ${ }^{10}$ tablets, ${ }^{10}$ personal computers, ${ }^{19,41}$ and visual display terminals. ${ }^{41}$ Personal technology use has been on the rise in the general population overall but especially in the younger active population. ${ }^{10,41}$ Measurement of Forward Head and Rounded Shoulder Posture

A common postural assessment tool is a plumb line, in which lateral, anterior, and posterior views can be monitored. During the assessment, the clinician examines specific anatomical structures and relation to the plumb line. In the lateral view forward head can be measured by examining the bodies of the cervical vertebra and the auditory meatus to see if the structures are bisected by the plumb line. In addition, rounded shoulders can also be monitored from the lateral view by examining the acromion process and whether it is bisected by the plumb line. Scapular positioning can be viewed from the posterior view by examining the distance between the scapular border and acromion process from the plumb line compared bilaterally, as well as the shoulder height. ${ }^{53}$ Individuals suffering from shoulder and cervical pain will be examined with a combination of clinical tests and measures not limited to signs and symptoms, range of motion, pain provocation tests, manual muscle tests, a plumb line, and/or diagnostic imaging. However, research has shown that the interrater reliability of the tests and measures was variable, adding additional subjectivity to the examination process. ${ }^{54}$

The $\mathrm{CV}$ angle is an objective measure for forward head posture that has been found to have success with test-retest and has shown that decreased CV angles correlate with increased 
cervical pain as well as a greater degree of forward head posture. ${ }^{37}$ (Table B1) The CV angle measures the angle between a horizontal line through the body of the $7^{\text {th }}$ cervical vertebra and a line from the $7^{\text {th }}$ cervical vertebra and the tragus of the ear. ${ }^{14}$ A 50-degree angle has been used in research as a reference angle; an individual with less than a 50-degree $\mathrm{CV}$ angle is considered to have a FHP. ${ }^{29}$ (Table B1) There have been a number of methods to measure the CV angle, each with varying reliability. (Table B1) Thigpen ${ }^{39}$ used reflective markers on the anatomical landmarks and measured the angle from a photograph whereas Ruivo ${ }^{29}$ used postural analysis software to compute the angle. Lau $^{37}$ used an electronic head posture instrument to digitally compute the $\mathrm{CV}$ angle. An objective measure for RSP is the acromion-table distance, which has shown a good to excellent inter-rater reliability. ${ }^{32,55}$ The acromion-table distance is an objective, static measurement where a patient is placed supine on a table in a relaxed position, and then the clinician measures the distance from the acromion process to the table the patient is laying on. ${ }^{55}$ Lee et al. ${ }^{19}$ also used the acromion to table distance and then correlated the distance to a pectoralis minor index. The index was measured from the origin to the insertion of the pectoralis minor. It was determined that there was a strong negative correlation between the amount of forward scapula through the acromion to table distance and the pectoralis minor index. ${ }^{19}$ (Table B1)

An additional RSP assessment is the use of the angle between a line from the $7^{\text {th }}$ cervical spinous process to the acromion process and a horizontal line through the acromion process - the shoulder angle. A 52-degree reference angle has been used; an individual with less than a 52degree shoulder angle are classified as having a rounded shoulder posture. ${ }^{29}$ (Table B1) Ruivo et $\mathrm{al}^{29}$ found a statistically significant difference across gender and CV angle; females in the study tended to have a greater FHP than males. Similar to the CV angle used in the studies by Cheung- 
Lau, ${ }^{37}$ Ruivo, ${ }^{29}$ and Ting Yip ${ }^{14}$ is the forward head angle (FHA) ${ }^{39}$ (Table B1) The FHA uses the tragus of the ear, the $\mathrm{C} 7$ vertebrae, and a vertical line through the $\mathrm{C} 7$ vertebrae. The angle was taken from the vertical line and a straight line that bisected the tragus and the $\mathrm{C} 7$ vertebrae. The study by Thigpen et al. ${ }^{39}$ also used the FSA to determine RSP. The angle was measured from the vertical line and a straight line that bisected the acromion process and the $\mathrm{C} 7$ vertebrae. The subjects underwent the postural assessment using the FSA and FHA to determine FHRSP. Those subjects with FHRSP had an average FHA of 51.9 degrees and a FSA of 57.7 degrees, while average angles for the subjects without FHRSP were 35.4 and 14.9 degrees, respectively. ${ }^{39}$ The last study ${ }^{37}$ assessed reliability and validity of an electronic head posture instrument (EHPI) to determine the CVA. (Table B1) Pins were placed on both the C7 vertebrae and the tragus of the ear and EHPI was adjusted to a virtual line between the two pins. Two clinicians measured the CVA separately and determined that the EHPI had high intrarater and interrater reliability for assessing CVA. ${ }^{37}$ A comparison of the varying methods to measure FHP and RSP is discussed in Table B1. (Table B1)

Measurement of Scapular Dyskinesis

The classifications of Scapular dyskinesis can be measured either statically or dynamically. (Table B1) One dynamic classification is a subjective examination where the clinician palpates the scapula, ensuring to monitor the medial and inferior scapular borders while the patient then abducts the arms simultaneously. The clinician palpates for any prominence that occurs during arm abduction on the inferior-medial, medial, and/or superior medial borders of the scapula. ${ }^{27}$ Further studies ${ }^{24-26}$ examined evaluation techniques for scapular dyskinesis and determined the interrater and intrarater reliability of similar techniques. (Table B1) $\mathrm{McClure}^{24}$ had the subjects use weights when performing shoulder flexion and frontal plane abduction while 
being videotaped. Two raters then analyzed the videotapes separately and any abnormal scapular motion was classified as normal, subtle, or obvious. The raters had a $75-82 \%$ agreement in regards to scapular dyskinesis classification for 30 subjects. ${ }^{24}$ (Table B1) In the Kibler ${ }^{25}$ study the subjects were videotaped while performing bilateral arm elevation in the scapular plane and bilateral arm abduction. Four clinicians initially determined interrater reliability by watching the recordings and classifying the scapular motion. Seventeen days later the recordings were shown to two of the original clinicians to determine intrarater reliability. A four-type classification system was used. Type I was inferior angle prominence during motion, type II was medial border prominence during motion, type III was superior border prominence during motion, and type IV was normal scapular motion. This classification system was similar but varied from the one used by Huang et al. ${ }^{27}$ (Table B1.) It was determined that there was a moderate level of agreement and reliability between the clinicians, both initially and 17 days later. ${ }^{25} \mathrm{Uhl}$ et al. ${ }^{26}$ assessed both the reliability and validity of a scapular dyskinesis classification system; two different classification systems were used. The 2-type system was whether scapular motion was normal or abnormal ${ }^{26}$ and the 4-type system was the same as used by Kibler. ${ }^{25,26}$ Two clinicians were blinded and assessed the motion of the subjects using both the 2 and 4 type systems. Both systems yielded moderate agreement, $61 \%$ for the 4 type and $79 \%$ for the 2 -type system, however the 4-type method had a higher specificity but a lower sensitivity than the 2-type system. In addition a 3-dimensional (3-D) kinematic analysis was performed to determine the validity of the two clinicians classification, which ranged from $45-66 \%$ for both systems. ${ }^{26}$ The motions by both $\mathrm{Uh}^{26}$ and $\mathrm{Kibler}^{25}$ did not use any weights as was used in the study by McClure. ${ }^{24}$ (Table B1) 
An adaptation of the classification system used by $\mathrm{McClure}^{24}$ was utilized by Butowicz ${ }^{38}$ in the form of a scoring system. (Table B1) The scoring system placed numerical values for normal (3), subtle (2), and obvious (1) scapular winging during weighted shoulder flexion and abduction, with pain during motion being scored as zero. ${ }^{38}$ It was suggested by Butowicz ${ }^{38}$ that the rater agreement would remain $75-82 \%$ since the scapular dyskinesis scoring system simply put numerical values on abnormal scapular motions described by McClure. ${ }^{24}$ The scoring system allows the measurement of scapular dyskinesis to be an objective measure, adding ease to the tracking of progression for patients. ${ }^{38}$

The static assessment is an objective measure where the inferior, lateral, and abduction displacement of the scapula is compared bilaterally by the clinician, while the patient is erect and arms adducted. Taking the vertical distance of the superior-medial scapular border and comparing it to the unaffected side measures the inferior displacement of the scapula. ${ }^{23}$ To measure the lateral displacement, the clinician calculated the difference in distance between the superior-medial scapula and the midline for the affected scapula and compared it to the unaffected scapula. ${ }^{23}$ The amount of scapular abduction is the angle between the vertical midline over the scapula and the medial border of the scapula. ${ }^{23}$

Table B1. Measurement Techniques for FHRSP and Scapular Dyskinesis

\begin{tabular}{|c|c|c|c|}
\hline Author & Purpose & Measurement & Results \\
\hline Thigpen et al. ${ }^{39}$ & $\begin{array}{l}\text { Compare scapular } \\
\text { kinematics and } \\
\text { muscle activity in } \\
\text { those individuals } \\
\text { with and without } \\
\text { FHRSP. }\end{array}$ & $\begin{array}{l}\text { Reflective markers on the C7 } \\
\text { vertebrae, tragus of the ear, } \\
\text { and acromion process where } \\
\text { used to measure the degree of } \\
\text { FH angle (FHA) and FS angle } \\
\text { (FSA). }\end{array}$ & $\begin{array}{l}\text { The average angles } \\
\text { of those classified } \\
\text { having FHRSP was } \\
51.9 \text { for FHA and } \\
57.7 \text { for FSA and } \\
\text { those classified as } \\
\text { not having FHRSP } \\
\text { had angles of } 35.4 \\
\text { and } 14.9 \text { for FHP } \\
\text { and RSP } \\
\text { respectively. }\end{array}$ \\
\hline
\end{tabular}


Lee et al. ${ }^{19}$

McClure et al. ${ }^{24}$

Kibler et al. ${ }^{25}$
Determine the relationship between a forward scapula (RSP) and pectoralis minor index.

Determine the interrater reliability of a new test to detect abnormal scapular motion.

Determine the
interrater and
intra-rater
reliability of the
clinical evaluation
for scapular
dyskinesis.

Determine the intra-rater reliability of the clinical evaluation dyskinesis.
Forward scapula was measured by the distance between the acromion and the wall while standing erect against the wall. Pectoralis minor index was measured from the origin (inferior medial edge of the coracoid process) to the insertion (caudal edge of the fourth rib adjacent to the sternum) of the pectoralis minor.

Subjects were videotaped from the posterior view while performing 5 repetitions of weight bilateral shoulder flexion and frontal plane abduction. 3 pairs of raters examined the videos and determined if abnormal scapular motion was present and if the right and left sides were normal, subtle, or obvious.

The subjects performed bilateral arm elevations in scaption and abduction while being videotaped. 2 physicians and 2 physical therapists reviewed the videotape initially for interrater reliability, and 1 additional physician and physical therapist 17 days later for intra-rater reliability. All of the clinicians classified the scapular dyskinesis as type IIV.
There was a strong negative correlation $(\mathrm{r}=-.89)$ between the degree of forward scapula and the pectoralis minor index.

The agreement between the pairs of raters ranged from $75-82 \%$ for the 30 subjects.

A moderate level of agreement and reliability was established between both the 2 initial physicians $(\kappa=.31)$ and 2 initial physical therapists $(\kappa=.42)$. There was also moderate intratester reliability for the additional physician $(\kappa=.59)$ and physical therapist $(\kappa=.49)$. 


\begin{tabular}{|c|c|c|c|}
\hline Uhl et al. ${ }^{26}$ & $\begin{array}{l}\text { Assess the } \\
\text { interrater } \\
\text { reliability and } \\
\text { validity of } 2 \\
\text { assessment } \\
\text { methods for } \\
\text { categorizing } \\
\text { scapular } \\
\text { dyskinesis while } \\
\text { quantifying the } \\
\text { asymmetry of } \\
\text { bilateral scapular } \\
\text { motion between } \\
\text { injured and non- } \\
\text { injured shoulders } \\
\text { using 3-D } \\
\text { kinematic analysis. }\end{array}$ & $\begin{array}{l}\text { Two clinicians were blinded } \\
\text { while assessing the subjects } \\
\text { during arm elevation in the } \\
\text { scapular and sagittal planes. } \\
\text { The clinicians classified each } \\
\text { subject into } 4 \text { types of scapular } \\
\text { motion, with types I-III being } \\
\text { abnormal and type IV being } \\
\text { normal and then } 2 \text { types of } \\
\text { scapular motion, abnormal and } \\
\text { normal. The subjects were then } \\
\text { fitted with a 3D tracking } \\
\text { device while performing arm } \\
\text { elevation in the sagittal and } \\
\text { scapular planes and compared } \\
\text { the 3D motion with the } \\
\text { clinicians' classification. The } \\
\text { 3D motion was used as the } \\
\text { reference standard. }\end{array}$ & $\begin{array}{l}\text { Using the 4-type } \\
\text { classification } \\
\text { system yielded a } \\
61 \% \text { agreement, } \\
\text { while the 2-type } \\
\text { yielded a } 79 \% \\
\text { agreement between } \\
\text { clinicians. The } \\
\text { accuracy of both } \\
\text { classification } \\
\text { methods ranged } \\
\text { from } 45-66 \% \text { while } \\
\text { the 4-type method } \\
\text { had a higher } \\
\text { specificity and } \\
\text { lower sensitivity } \\
\text { overall than the 2- } \\
\text { type method. }\end{array}$ \\
\hline Ruivo et al. $^{29}$ & $\begin{array}{l}\text { Determine the } \\
\text { interrater and } \\
\text { intra-rater } \\
\text { reliability of } \\
\text { photographic } \\
\text { measurements of } \\
\text { sagittal postures, } \\
\text { quantify the } \\
\text { posture for the } \\
\text { head and shoulders } \\
\text { in the sagittal } \\
\text { plane and analyze } \\
\text { for sex differences. }\end{array}$ & $\begin{array}{l}\text { Reflective markers were } \\
\text { placed on the tragus of the ear, } \\
\text { the spinous process } \mathrm{C} 7 \text {, and } \\
\text { the midpoint of the humerus. } \\
\text { A photographic was taken and } \\
\text { postural analysis software was } \\
\text { used to measure the cervical } \\
\text { and shoulder angles. A } \\
\text { separate sample was measured } \\
\text { for the reliability study. }\end{array}$ & $\begin{array}{l}\text { Both measurements } \\
\text { reported good } \\
\text { reliability, . } 78 \text { for } \\
\text { the shoulder angle } \\
\text { and .66 for the } \\
\text { cervical angle. } \\
\text { Females had a } \\
\text { statically significant } \\
\text { smaller cervical } \\
\text { angle, ( } 46.55 \text { vs. } \\
48.44) \text { but the } \\
\text { shoulder angle } \\
\text { (51.09 vs. } 51.9) \text { was } \\
\text { not significantly } \\
\text { different than } \\
\text { males, meaning } \\
\text { more of a forward } \\
\text { head posture but } \\
\text { rounded shoulder } \\
\text { posture. }\end{array}$ \\
\hline
\end{tabular}




\begin{tabular}{|c|c|c|c|}
\hline $\begin{array}{l}\text { Cheung-Lau et } \\
\text { al. }{ }^{37}\end{array}$ & $\begin{array}{l}\text { Assess the } \\
\text { reliability and } \\
\text { validity of the } \\
\text { electronic head } \\
\text { posture instrument } \\
\text { (EHPI) in } \\
\text { measuring the } \\
\text { craniovertebral } \\
\text { angle in patients } \\
\text { with and without a } \\
\text { history of neck } \\
\text { pain. }\end{array}$ & $\begin{array}{l}\text { A pin marker was placed on } \\
\text { the most prominent point of } \\
\text { the subjects' } \mathrm{C} 7 \text { vertebra and } \\
\text { the tragus of the ear. A virtual } \\
\text { line was drawn between the } \\
\text { two points and then the EHPI } \\
\text { was adjusted to the virtual line } \\
\text { and the CV angle was } \\
\text { measured. Two clinicians } \\
\text { measured each subject } \\
\text { separately. }\end{array}$ & $\begin{array}{l}\text { There was a high } \\
\text { intra-rater (ICC .86- } \\
.94) \text { and interrater } \\
(.85-.91) \text { reliability } \\
\text { in measuring the } \\
\text { CV angle in both } \\
\text { groups. Those } \\
\text { patients with a } \\
\text { history of neck pain } \\
\text { had a smaller CV } \\
\text { angle than those } \\
\text { with no history of } \\
\text { neck pain. }\end{array}$ \\
\hline Butowicz $^{38}$ & $\begin{array}{l}\text { Describe a novel } \\
\text { comprehensive } \\
\text { performance-based } \\
\text { movement system- } \\
\text { screening tool } \\
(\mathrm{MSST}) \text {. }\end{array}$ & $\begin{array}{l}\text { Subjects performed five } \\
\text { repetitions of bilateral } \\
\text { weighted shoulder flexion and } \\
\text { abduction while the researcher } \\
\text { examined and scored scapular } \\
\text { motion. The scoring used was } \\
3 \text { for normal scapular motion, } \\
2 \text { for subtle scapular winging } \\
\text { in } 3 / 5 \text { trials, } 1 \text { for obvious } \\
\text { scapular winging in } 3 / 5 \text { trials, } \\
\text { and } 0 \text { for pain during } \\
\text { movement. }\end{array}$ & $\begin{array}{l}\text { The researcher used } \\
\text { an adaptation of the } \\
\text { classification } \\
\text { system used by } \\
\text { McClure }{ }^{24} \text { and thus } \\
\text { the } 75-82 \% \text { range } \\
\text { of agreement was } \\
\text { utilized. }\end{array}$ \\
\hline
\end{tabular}

Treatment of Forward Head and Rounded Shoulder Posture

A FHP is associated with a tightness of the posterior cervical muscles and a weakness in the anterior cervical flexor muscles. This is caused by the neck to be constantly placed in a lower cervical flexion and upper cervical extension posture. Treatment and rehabilitation programs for FHP have focused on strengthening the deep cervical flexor muscles to correct the postural deviation. ${ }^{30}$ Ruivo et $\mathrm{al}^{29}$ utilized exercises that aimed at improving both FHP and RSP by using a combination of stretching and strengthening exercises whereas Gupta et $\mathrm{al}^{30}$ used only strengthening exercises for deep cervical flexors. Both studies exhibited a decrease in outcome measures, FHP and RSP for the Ruivo et $\mathrm{al}^{29}$ study and FHP, visual analogue scale, and neck disability index for the Gupta et $\mathrm{al}^{30}$ study. An exercise consistently used for strengthening the 
deep cervical flexors is the chin tucks exercise. The chin tucks exercise focuses on maintaining proper cervical posture by activating the deep cervical flexors. (Table B2) The use of an exercise protocol consisting of external rotation, prone horizontal abduction with external rotation, Y to I exercise, and chin tucks as well as PM, SCM, and levator scapulae stretches had a statistically significant decrease in CVA and further FHP. ${ }^{29}$ (Table B2) Research ${ }^{30}$ has shown that a deep cervical flexor-training program has decreased the amount of FHP in patients as well as pain and score on a neck disability index (NDI). The deep cervical flexor-training group was compared to isometric training as a control group, of which the control group had a decrease in pain and score on the NDI but did not have a statistically significant improvement of FHP as the intervention group did. ${ }^{30}$

A rounded shoulder posture involves a protracted scapula and then an anteriorly tilted coracoid process as a result. Attached to the coracoid process is the pectoralis minor, which has been found as a major cause of RSP by pulling on the coracoid process and subsequently the scapula by being hypertonic. ${ }^{31}$ Due to the altered positioning of the scapula, the force couple between the pectoralis minor and the lower trapezius and serratus anterior muscles is distorted, causing a decrease in strength and activation of the lower trapezius and serratus anterior muscles. ${ }^{31}$ A treatment and rehabilitation program focused on stretching the pectoralis minor exhibited a statistically significant decrease in the amount of RSP after a two-week intervention. ${ }^{31}$ Stretches for the pectoralis minor can be performed actively without the assistance of a clinician or passively with a clinician. The active pectoralis minor stretch is the corner stretch, where the patient leans into the corner of a wall with their arms being pushed into horizontal abduction. (Table B2) The passive stretch involves the clinician rotating the patient's neck and depressing the shoulder to place the pectoralis minor on a stretch. (Table B2) Wong et $\mathrm{al}^{31}$ examined the 
effects of a self-stretch of the PM in conjunction with soft tissue mobilization of the PM and compared it to a passive placebo touch of the PM and self stretch of the PM. Results determined there was no significant difference between groups. ${ }^{31}$ (Table B2) Further research has shown that the use of a scapular posterior tilting exercise in addition to a pectoralis minor stretch was effective in reducing RSP. ${ }^{19}$ Another intervention in the study by Lee et al. ${ }^{19}$ incorporated a postural shoulder brace with the scapular posterior tilting exercise also reduced the amount of RSP in participants. (Table B2) The postural shoulder brace acted as a proprioceptive biofeedback to the participant to retract their shoulders for optimal posture. Treatment of Scapular Dyskinesis

Scapular dyskinesis defined as an alteration in the kinematics of the scapula itself. The altered kinematics distort force-couples and length-tension relationships, therefore a rehabilitation program for Scapular dyskinesis must incorporate exercises designed to regain neuromuscular control of scapular stabilization muscles. ${ }^{23}$ Scapular dyskinesis is associated with a postural deviation such as a rounded shoulder or forward head posture. ${ }^{27,32}$ The rehabilitation and treatment of scapular dyskinesis focuses on stretching the pectoralis minor and posterior glenohumeral joint capsule, while strengthening and regaining control of the scapular stabilizers using closed kinetic chain exercises. ${ }^{23}$ Strengthening exercises for scapular stabilizers involve scapular depression, elevation, protraction, and retraction, as well as Y's to I's. ${ }^{23,29}$ The Y's to I's exercise involves the patient retracting both scapulae while the shoulders are abducted 90 degrees and elbows flexed 90 degrees. The elbows are then brought into extension while the shoulder continues into further abduction above the head while keeping the scapula retracted. ${ }^{29}$ Two studies had a focus on scapular dyskinesis, Lee et a ${ }^{19}$ and Burkhart et al ${ }^{23}$ Lee et $\mathrm{al}^{19}$ specifically examined the muscle activity of the LT and SA muscles that remain important 
segments of the scapular force couples. The study by Burkhart et $\mathrm{al}^{23}$ related to professional baseball pitchers whom exhibited pain while throwing as well as scapular dyskinesis and brought the pitchers through a rehabilitation program that included exercises as well as an intervalthrowing program. The exercises focused on correcting the length tension relationships on the shoulder and scapula while also inducing correct biomechanics and neuromotor control while throwing. Lee et $\mathrm{al}^{19}$ was successful at increasing the activity LT and SA while the subjects in the Burkhart et $\mathrm{al}^{23}$ study were able to throw pain free after the four month rehabilitation program due to a decrease in scapular dyskinesis. (Table B2) The most important concept with scapular dyskinesis rehabilitation is adherence to a maintenance exercise program focused on scapular stabilization and neuromuscular control. It has been shown the overhead athletes with pain during throwing, were all returned to pain-free throwing within 3-4 months of undergoing a scapular strengthening program and all but nine athletes remained pain-free after 1 year. Those nine athletes admitted to non-adherence to the maintenance program. ${ }^{23}$

Treatment of scapular dyskinesis involves postural correction as well as neuromuscular reeducation. The exercises used the Lee et al. ${ }^{19}$ studied were the scapular posterior tilt exercise and a PM stretch. The study examined the effects of the scapular posterior tilt exercises in conjunction with either the PM stretch or a shoulder brace for increasing LT and SA activity and decreasing RSP. The study by Wong ${ }^{31}$ utilized the PM stretch as well as soft tissue mobilization for treatment of RSP and found no significant difference between the treatment and placebo group. The study by Ruivo ${ }^{29}$ evaluated the effect of exercises on correcting FHP and protracted scapulae, which only corrects the postural but not the proprioceptive deficits also associated with scapular dyskinesis. Each study involved an exercise program but the aim of the exercises 
varied, whether the correction of FHP and RSP was the goal or the neuromuscular reeducation,

or both. ${ }^{19,29,31}$

Table B2. Treatment techniques for FHRSP and Scapular Dyskinesis

\begin{tabular}{|c|c|c|c|}
\hline Author & Purpose & Intervention & Results \\
\hline Ruivo et al. ${ }^{29}$ & $\begin{array}{l}\text { Evaluate the } \\
\text { effects of both } \\
\text { a 32-week } \\
\text { training } \\
\text { program } \\
\text { followed by a } \\
\text { 14-week } \\
\text { detraining } \\
\text { period on FHP } \\
\text { and protracted } \\
\text { scapulae on } \\
\text { Portuguese } \\
\text { adolescents. }\end{array}$ & $\begin{array}{l}\text { Supervised exercises were } \\
\text { performed twice a week at the end } \\
\text { of each physical education class. } \\
\text { The four strengthening exercises } \\
\text { used were side lying external } \\
\text { rotation, prone horizontal } \\
\text { abduction with external rotation, } \\
\text { Y to I exercise, and chin tucks. } \\
\text { The three stretching exercises } \\
\text { used were one-sided unilateral } \\
\text { self-stretch of the PM, static SCM } \\
\text { stretch, and a static levator } \\
\text { scapulae stretch. Strengthening } \\
\text { exercises started with } 2 \text { set of } 10 \\
\text { and progressed, while stretching } \\
\text { exercises started with } 2 \text { sets of } 30 \text { - } \\
\text { second hold. }\end{array}$ & $\begin{array}{l}\text { There was a significant } \\
\text { decrease in CV angle } \\
\text { and sagittal head angle } \\
\text { for the experimental } \\
\text { group with } p<.05 \text {, } \\
\text { while there was no } \\
\text { decrease in the control } \\
\text { group. This shows that } \\
\text { the experimental group } \\
\text { had a decrease in the } \\
\text { amount of FHP and } \\
\text { protracted scapulae } \\
\text { following the } \\
\text { intervention. }\end{array}$ \\
\hline Gupta et al. ${ }^{30}$ & $\begin{array}{l}\text { Determine and } \\
\text { compare the } \\
\text { effect of deep } \\
\text { cervical flexor } \\
\text { (DCF) training } \\
\text { on FHP, visual } \\
\text { analogue scale } \\
\text { (VAS) and } \\
\text { neck disability } \\
\text { index (NDI) } \\
\text { compared with } \\
\text { conventional } \\
\text { isometric } \\
\text { training (CIT) } \\
\text { in dentists with } \\
\text { chronic neck } \\
\text { pain. }\end{array}$ & $\begin{array}{l}\text { Baseline FHP, VAS, and NDI } \\
\text { measurements were taken. The } \\
\text { experimental group was brought } \\
\text { through a supervised DCF } \\
\text { training for four weeks while the } \\
\text { control group was brought } \\
\text { through a supervised CIT for four } \\
\text { weeks. }\end{array}$ & $\begin{array}{l}\text { The experimental group } \\
\text { had a significant } \\
\text { decrease in FHP, VAS, } \\
\text { and NDI ( } \mathrm{p}=.000 \text { for } \\
\text { all three), while the } \\
\text { control group did not } \\
\text { have a significant } \\
\text { decrease for FHP ( } \mathrm{p}= \\
\text {.164) but did for VAS } \\
\text { and NDI ( } \mathrm{p}=.000 \text { for } \\
\text { both). FHP was } \\
\text { significantly improved } \\
\text { in the experimental } \\
\text { group but not the } \\
\text { control group, while } \\
\text { VAS and NDI } \\
\text { improved in both. }\end{array}$ \\
\hline
\end{tabular}


Lee et al. ${ }^{19}$

Wong et al. ${ }^{31}$
To determine

which

intervention, the scapular posterior tilting exercise (SPT), SPT with a PM stretch, or SPT with a shoulder brace is most effective in decreasing RSP and PM index, while increasing LT and SA muscle activation.
Every subject performed one of the three interventions in random order, then the RSP, PM index, and LT/SA activity was measured. There was a 12minute waiting period before the next intervention and measurement was taken. Every subject performed all three interventions in random order.
There was a significant decrease in RSP for the SPT with a PM stretch $(\mathrm{p}<.05)$ and SPT with a shoulder brace group $(\mathrm{p}<.05)$. There was a significant decrease in PM index for the SPT with a PM stretch (p $=.017$ ) and SPT with a shoulder brace group ( $\mathrm{p}$ $=.004$ ). There was a significant increase in LT activity following the SPT with PM stretch (.0009) but not the SPT or SPT with a shoulder brace. There was no significant difference in SA activity between all three interventions. The use of SPT and SPT brace shows effectiveness in decreasing RSP and increasing LT activity.

There was no significant difference between the intervention and placebo group for RSP and muscle activity of the LT $(\mathrm{p}<.05)$ There was an improvement in the intervention group after the first treatment compared to the placebo but the difference was not significant. 


\begin{tabular}{|c|c|c|c|}
\hline Burkhart et al. ${ }^{23}$ & $\begin{array}{l}\text { To determine if } \\
\text { a rehabilitation } \\
\text { program } \\
\text { consisting on } \\
\text { scapular } \\
\text { muscle } \\
\text { rehabilitation } \\
\text { and a throwing } \\
\text { interval } \\
\text { program is } \\
\text { effective in } \\
\text { treating SICK } \\
\text { scapula in } \\
\text { professional } \\
\text { baseball } \\
\text { pitchers. }\end{array}$ & $\begin{array}{l}\text { Professional baseball pitchers } \\
\text { who scored on a } 20 \text {-point } \\
\text { assessment for SICK scapula and } \\
\text { with a painful throwing shoulder } \\
\text { were included. The program } \\
\text { included both strengthening and } \\
\text { stretching exercises. The } \\
\text { stretching exercises focused on a } \\
\text { foam roller stretch of the } \\
\text { pectoralis minor and an assisted } \\
\text { posterior glenohumeral capsule } \\
\text { stretch ("sleeper stretch"). The } \\
\text { strengthening exercises included } \\
\text { were closed kinetic chain scapular } \\
\text { protraction, retraction, elevation, } \\
\text { depression, and internal and } \\
\text { external rotation against a mirror, } \\
\text { which progressed to open kinetic } \\
\text { chain first without weight, then } \\
\text { with a } 2 / 3 \text { lb. weight. The last } \\
\text { exercises used were Blackburn } \\
\text { exercises, seat push-ups, and } \\
\text { rowing exercises both standard } \\
\text { and low row. }\end{array}$ & $\begin{array}{l}\text { All } 96 \text { patients included } \\
\text { had symptom and pain } \\
\text { free throwing after } 4 \\
\text { months following the } \\
\text { program. However, } 9 \\
\text { of the } 96 \text { had a } \\
\text { reoccurrence in } \\
\text { symptoms and pain } \\
\text { after the } 4 \text { months and } \\
\text { admitted to non- } \\
\text { adherence to the } \\
\text { maintenance program. }\end{array}$ \\
\hline
\end{tabular}

Kinesiotape

Kinesiotape was founded in the late 1970's by Kenzo Kase and is an elastic adhesive tape with multiple clinical uses. ${ }^{34}$ Uses for KT include, but are not limited to neuromuscular reeducation, inflammation and swelling reduction, promoting circulation, and proprioception. ${ }^{34,35}$ Recently KT has risen in popularity due to its use by professional athletes with national television exposure. KT for the use of proprioception is based on stimulating superficial sensory nerves that are reacting to the KT as the stimuli. ${ }^{33}$ A number of studies ${ }^{56-58}$ have shown that Kinesiotape is ineffective in terms of neuromuscular performance, ${ }^{56}$ muscular facilitation and inhibition, ${ }^{57}$ and mechanical correction in terms of joint instability, muscle strains, and ligamentous sprains. ${ }^{58}$ Oliveira et al. ${ }^{56}$ examined the effects of KT on quadriceps neuromuscular performance and balance for individuals 12-17 weeks post operation for an anterior cruciate 
ligament (ACL) reconstruction. Following the intervention of KT application, none of the variables analyze showed any significant improvement whether pre and post test or been experimental and control groups. ${ }^{56}$ The use of KT for performance enhancement was assessed by Cai et al.$^{57}$ The study compared the neuromuscular activity for the wrist extensor muscles and maximal grip strength on subjects. The interventions included KT for muscular facilitation, KT for muscular inhibition, and no KT for the control group. No significant differences were found between groups for either of the variables in the study. ${ }^{57}$

Further research ${ }^{35}$ has shown that KT is best used in conjunction with physical therapy for optimal results in regards to proprioception and muscular endurance. ${ }^{59}$ It has been found that KT can also increase ROM and decrease pain associated with shoulder pathologies, when performed in combination with manual therapy. ${ }^{43}$ There have been case reports ${ }^{36,58}$ that have demonstrated effectiveness of KT on upper back pain and treatment for an acute hamstring strain. One study ${ }^{36}$ found a decrease in RSP, as well as upper back pain, in a sedentary worker following a 6-week KT application period. However, this study ${ }^{36}$ was confined as a case report with only one female sedentary worker and the results are not generalizable. The application of $\mathrm{KT}$ in the case report is similar to the pattern that will be utilized in this study. ${ }^{36}$ (Table C9) Outside of the case report by Hwang-Bo et al. there has been no research examining the use of KT for correction of FHP, RSP, and scapular dyskinesis. Guner and Alsancak assessed a 19year-old patient with an acute hamstring strain and the effects of KT for muscular facilitation for the hamstring. The results were that the patient had a significant increase in range of motion and cadence during gait. ${ }^{58}$ Overall, there is a lack of viable research on the effectiveness of KT, specifically in terms of proprioception for postural control.

Summary 
Neck and shoulder pain are a common pathology within the athletic and general population. The recent increase in use of smartphones, computer, and visual display terminals has been a fundamental cause of neck and shoulder pain. The biomechanical underlying factors to neck and shoulder pain have been postural deviations such as FHP and RSP, as well as associated scapular dyskinesis. FHP places stress onto the cervical flexors and overuse onto the cervical extensors, while RSP alters the biomechanics of the glenohumeral joint when the scapula and further the acromion process anteriorly tilt. Reliable, objective and subjective findings can be measured for FHRSP as well as scapular dyskinesis. FHRSP is measured through the FHA and the CVA, while a scoring system is used to measure scapular dyskinesis. The treating for postural distortions involves a combination of stretching, strengthening, and regaining neuromuscular control for the shoulder, upper back, and cervical region. Treatment typically consists of manual therapy and rehabilitation exercises that focus on correcting the postural deviations. More recently, KT has been used as a possible means of conjunction therapy, although the research and results have been limited. 
APPENDIX C

ADDITIONAL METHODS

C1. Consent Information and HIPAA Form

\section{Only Minimal Risk \\ Consent Information and HIPAA Form}

Principal Investigator

Department

Protocol Number

Study Title

Shoulder Post

Co-Investigator(s)

Sponsor (if any)
Michelle Sandrey, PhD, ATC

College of Physical Activity and Sport Sciences

Click here to enter text.

The Effects of a Kinesiotape Intervention on Forward Head/Rounded

\section{Contact Persons}

In the event you experience any side effects or injury related to this research, you should contact PI Dr.

Michelle A. Sandrey, PhD, ATC at (304) 293-0870 or at msandrey@mail.wvu.edu or Co PI Luke Klawiter, ATC, CES at (616)-340-5291 or luklawiter@mix.wvu.edu.

For information regarding your rights as a research subject, to discuss problems, concerns, or suggestions related to the research, to obtain information or offer input about the research, contact the Office of Research Integrity and Compliance at (304) 293-7073.

In addition if you would like to discuss problems, concerns, have suggestions related to research, or would like to offer input about the research, contact the Office of Research Integrity and Compliance at 304-293-7073.

\section{Introduction}

You have been asked to participate in this research study, which has been explained to you by Luke Klawiter, ATC, CES. This study is being conducted by principle investigator Dr. Michelle A. Sandrey, PhD, ATC and Co-Investigator Luke Klawiter, ATC, CES in the College of Physical Activity and Sport Sciences at West Virginia University. This study is being completed for the partial fulfillment of the requirements for the degree of Master of Science in Athletic Training.

\section{Purpose(s) of the Study}

The purpose of this study is to determine whether an exercise or kinesiotape intervention is an effective treatment for forward head/rounded shoulder posture and scapular winging.

\section{Description of Procedures}


This study involves completing a demographic questionnaire with injury history for inclusion criteria, partaking in pre and posttest measurements, and being placed in one of two intervention groups. You do not have to answer all the questions in the questionnaire. You will have the opportunity to see the questionnaire before signing this consent form.

The pre and post-test measurements will involve placing reflective markers on you for predetermined body structures where a still photograph will be taken using an iPad. For the measurement of your forward head angle and the forward shoulder angle, markers will be placed on the spinous process of your $\mathrm{C} 7$ vertebrae, the middle of your ear on one side of your body, and the acromion process (tip of the shoulder) of the your shoulder on the same side of the body as the ear already marked. The still photograph will then be taken and the markers will be removed. You will then stand in a relaxed position, with your shirt removed (with a sports bra on for females), while the examiner observes your medial and superior scapular borders from a posterior view. You will then perform five repetitions of shoulder abduction (raising your shoulders from your side) as the examiner scores your scapulae (shoulder blades). Then you will perform three repetitions of shoulder flexion as the examiner again scores your scapulae.

You will be assigned to one of two intervention groups or a control group. The control group will not participate in any intervention during a 4-week period rather will be measured pre and post the 4-week period. If you are assigned to one of the intervention groups kinesiotape will be applied to the your back and shoulder for a duration five days and then the tape will be removed for two days. You will then be reapplied with tape the following week and repeat that process for four consecutive weeks. The other invention group will involve you being led through a series of strengthening and stretching exercises one time a week as well as adhering to an at-home exercise plan for a duration of four weeks. At the conclusion of the four-week period, the post-test measurements will be taken for all three groups.

\section{Discomforts}

There are no known or expected risks from participating in this study, except some soreness related to the pre/post tests or the intervention such as allergic reaction to the kinesiotape. If an allergic occurs, the coinvestigator will treat properly within reason, with a possible referral. Any fees incurred due to the referral will be at your own expense. You will also be removed from the study if an allergic reaction occurs.

\section{Alternatives}

You do not have to participate in this study. You may withdraw at any time with no penalty.

\section{Benefits}

You may not receive any direct benefit from this study. However, this study procedures and results may help aid in other research. It could help determine if kinesiotape or the exercises are an effective treatment for improper posture and scapular dyskinesis. The knowledge gained from this study may eventually benefit others.

\section{Financial Considerations}


There will be no payments made for participation in this study. There is no cost to participants in this study. You will not earn extra credit for participating in this study, nor will you be penalized academically for not participating.

\section{Confidentiality}

Any information about you that is obtained as a result of your participation in this research will be kept as confidential as legally possible. Your research records and test results, just like hospital records, may be subpoenaed by court order or may be inspected by the study sponsor or federal regulatory authorities without your additional consent. Audiotapes or videotapes will be kept locked up and will be destroyed as soon as possible after the research is finished. In any publications that result from this research, neither your name nor any information from which you might be identified will be published without your consent.

\section{HIPAA}

We know that information about you and your health is private. We are dedicated to protecting the privacy of that information. Because of this promise, we must get your written authorization (permission) before we may use or disclose your protected health information or share it with others for research purposes.

You can decide to sign or not to sign this authorization section. However, if you choose not to sign this authorization, you will not be able to take part in the research study. Whatever choice you make about this research study will not have an effect on your access to medical care.

USE AND DISCLOSURE COVERED BY THIS AUTHORIZATION. DO NOT SIGN A BLANK FORM. You or your authorized representative should thoroughly read the information below before signing this form. This form will authorize the following person(s), class(es) of persons, and/or organization(s) to disclose, use, and receive the information: WVU, Michelle A. Sandrey, PhD, ATC, Luke Klawiter, ATC, CES. The research site(s) carrying out this study includes WVU. If, during the course of the research, the institution listed above merges with, or is purchased by, another company or institution, this authorization to use or disclose protected health information in the research will extend to the successor, company, or institution. A self-reported demographic history that includes information on height, weight, past medical history of any upper extremity, lower extremity, or spine injury is included in this study.

SPECIFIC UNDERTANDINGS. By signing this research authorization form, you give permission for the use and/or disclosure of your protected health information described above. The purpose for the uses and disclosures you are authorizing us to carry out the research study explained to you during the informed consent process. It is also to ensure that the information relating to the research is available to all parties who may need it for research purposes. Your protected health information may be used as necessary for you research related treatment. This information may be redisclosed or used for other purposes if a recipient described in this form is not required by law to protect the privacy of the information. You have a right to refuse to sign this authorization if you do not sign this form. If you sign this authorization, you will have the right to cancel at any time, except to the extent that WVU has already taken action based upon your authorization or needs information to complete analysis and reports of data for this research study. This authorization will expire six months from today unless you cancel this sooner. To cancel this authorization, please write to the Principal Investigator, Michelle A. Sandrey, PhD, ATC at: West Virginia University, PO Box 6116, Morgantown, WV 26506. If you cancel this authorization, any information that was collected already for this study cannot be withdrawn. You will NOT be allowed to see or copy the information described on this form as long as the research is in progress, but you have a right to see and copy the information upon completion of the research in 
accordance with hospital policies. You have a right to receive a copy of this form after you have signed it. In any publications that result from this research, neither your name nor any information from which you might be identified will be published without your consent.

\section{Voluntary Participation}

Participation in this study is voluntary. You are free to withdraw your consent to participate in this study at any time. Refusal to participate or withdrawal will not affect your class standing or grades and will involve no penalty to you. Refusal to participate or withdrawal will not affect your future care, or your status in the athletic training program at West Virginia University. If an injury were to occur during the study, Luke Klawiter, ATC, will provide the necessary first aid. If a referral is necessary, I understand there will be at your expense. You have been given the opportunity to ask questions about the research, and you have received answers concerning areas you did not understand.

Upon signing this form, you will receive a copy.

I willingly consent to participate in this research.

\section{Signatures}

Signature of Subject

Printed Name

Date

Time

The participant has had the opportunity to have questions addressed. The participant willingly agrees to be in the study.

Signature of Investigator or Co-Investigator

Printed Name

Date

Time 
Table C2. Subject Questionnaire

Subject Number:

Age:

Gender: (Circle One) Male / Female

On average, how hours per day do you spend on your cellphone/smartphone?

On average, how hours per day do you spend on your computer/tablet?

Year in School: (Circle One) Freshman / Sophomore / Junior / Senior

Are you currently a Prospective Athletic Training Student (PATS) or a Curriculum Athletic Training Student (CATS)? (Circle One) Yes / No

Height:

Weight:

1) Have you had a history of upper body injury within the past six months that has required medical attention? If so, what was the diagnosis?

a) Shoulder?

b) Neck?

c) Head?

2) Are you currently receiving any type of therapy or treatment for any of the above injuries? (If no injuries, put N/A)

3)Are you currently taking any medications that may affect your balance or coordination?

4) Do you have any allergies to tape or adhesives? (Circle One) Yes / No / Unsure 
Step 1. The subject will stand in a relaxed, standing position, while the examiner marks the spinous process of the $7^{\text {th }}$ cervical vertebra and the tragus of the ear on one side of the body with a reflective marker.

Step 2. An Ipad will be used to take a still picture of the patient from a lateral view.

Step 3. The Hudl app on the Ipad will be used to determine the angle between a line going through the $7^{\text {th }}$ cervical vertebrae and the tragus of the ear and a line along the horizontal at the $7^{\text {th }}$ cervical vertebrae.

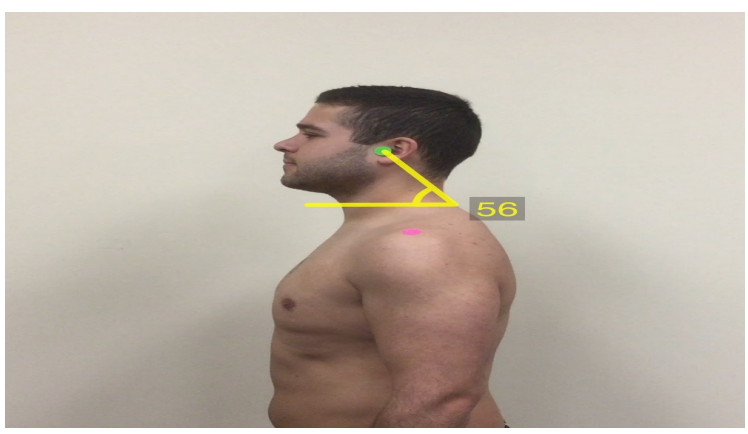

Table C4. Shoulder Angle ${ }^{19,29,39}$

Step 1. The subject will stand in a relaxed position, while the examiner marks the spinous process of the $7^{\text {th }}$ cervical vertebra and the midpoint of the shoulder on one side of the body with a reflective marker.

Step 2. An Ipad will be used to take a still picture of the patient from a lateral view.

Step 3. The Hudl app on the Ipad will be used to determine the angle between a line going through the $7^{\text {th }}$ cervical vertebrae and the midpoint of the shoulder and a line along the horizontal at the midpoint of the shoulder. 
Step 1. The subject will stand in a relaxed position, with their shirt removed (females will remain in a sports bra).

Step 2. An Ipad will be used to take a video recording of the patient to be analyzed at a later time.

Step 3. The subject will perform 5 repetitions of weighted shoulder abduction with a 2 lb weight.

Step 4. The subject will then perform 5 repetitions of weighted shoulder flexion with a $2 \mathrm{lb}$ weight.

Step 5. The examiner will score each scapula using a 0-3 scale.

(0: pain was present during the movement. 1: if there was obvious winging or dysrhythmia observed on $3 / 5$ trials in either flexion or abduction. 2: if there was subtle winging or dysrhythmia observed on 3/5 trials in either flexion or abduction. 3 : if no winging or dysrhythmia was observed. The maximum point total available is 6.$)^{38}$

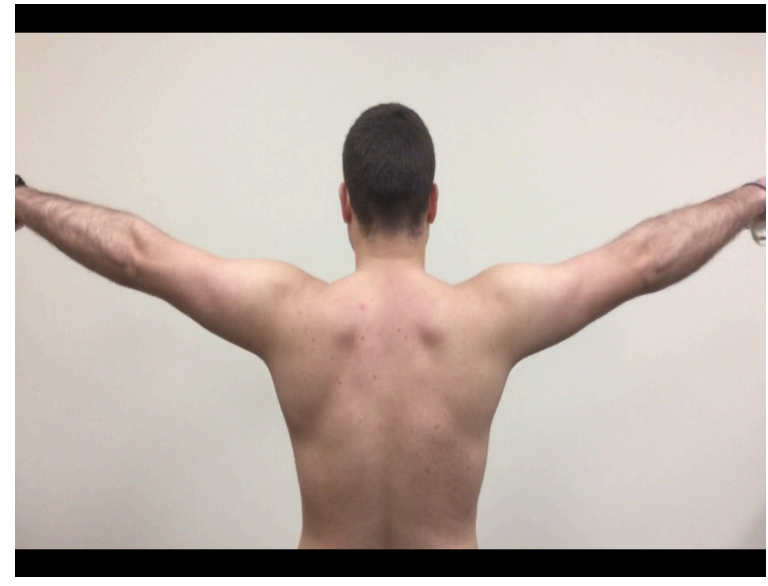


Table C6. Data Collection Sheet

Subjects Number:

Weight:

Height:

Data Collection Sheet for CV angle and Shoulder Angle, and Scapular Dyskinesis Measurement

Trial: Pre-test / Post-test

\section{Craniovertebral Angle}

Left:

Shoulder Angle

Left:

\section{Scapular Dyskinesis Scoring}

Right:

Left: 
$\underline{\text { Table C7. Exercise Intervention Protocol }}{ }^{19,23,29-31}$

A.

Home Exercise Protocol:

Exercise

Chin Tucks

Y's to I's

Wall Washes

Upright Scapula Punches

Corner Stretch

Static Sternocleidomastoid Stretch $\underline{\text { Sets }}$

3 sets

2 sets

3 sets/each arm

3 sets

3 sets

3 sets

$\underline{\text { Sets }}$

1 set/diagonal

2 sets

3 sets

3 sets

\section{Repetitions}

10 repetitions; 5 second hold

10 repetitions

10 repetitions

10 repetitions; 3 second hold

30 second hold

30 second hold

B.

Exercise

Scapular PNF

Y's to I's with chin tuck

Passive Sternocleidomastoid Stretch

Passive Pectoralis Minor Stretching

s

To fatigue
10 repetitions
30 seconds

30 seconds

30 seconds

If progression is necessary based on correct completion of exercises, a set will be added to the exercise. 


\section{Chin Tucks}
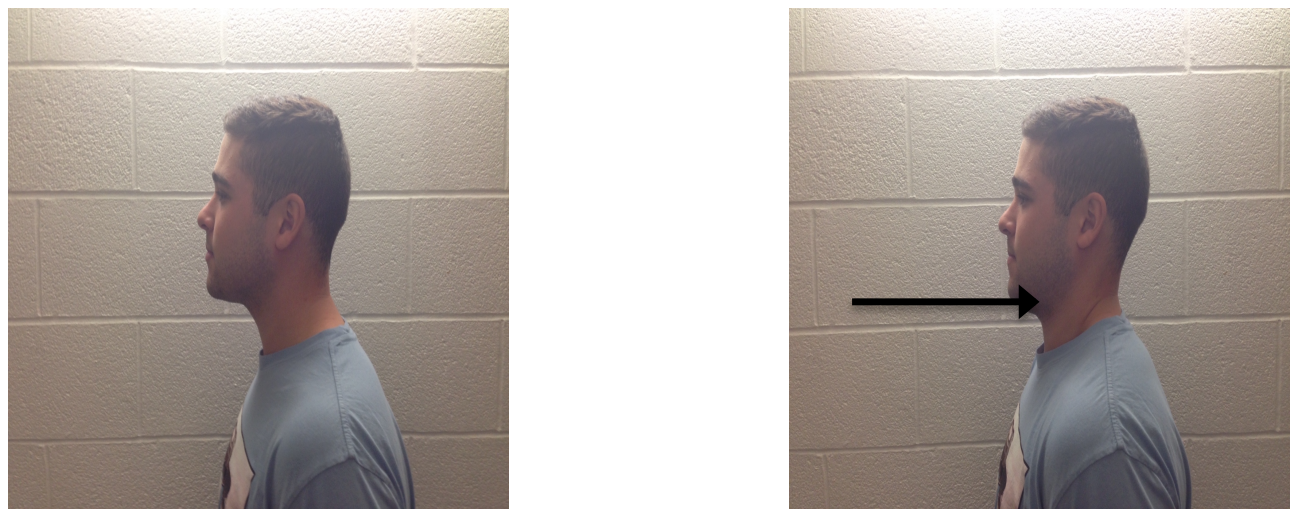

1. The subject will stand erect in a relaxed position

2. The subject will then tuck the chin and hold, then return to the starting position

Y's to I's
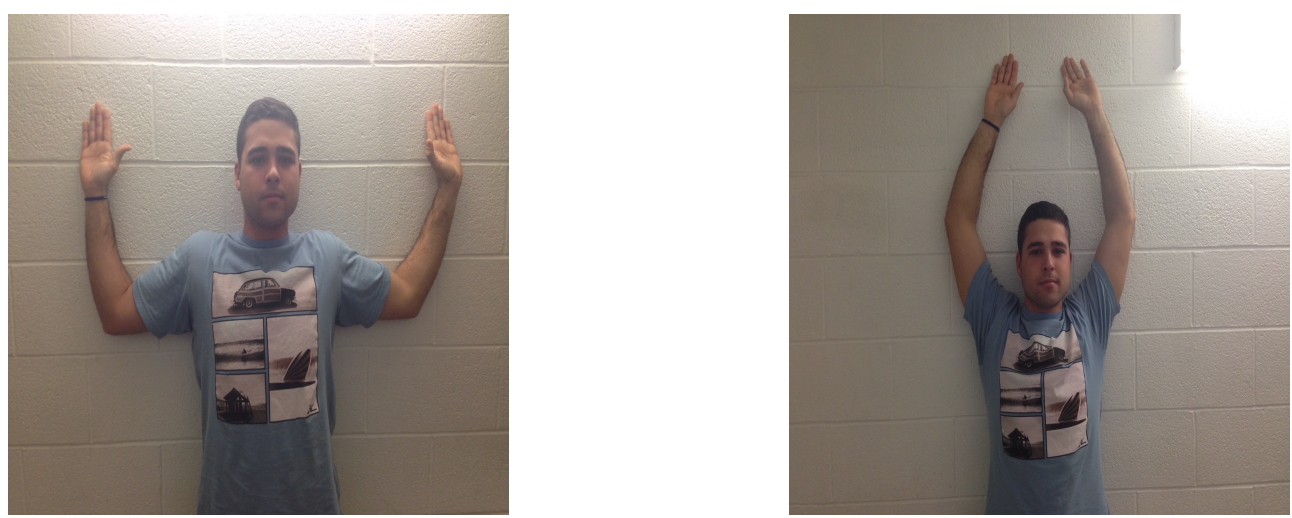

1. The subject will begin with their back against a wall

2. The subject will horizontally abduct the shoulders to 90 degrees and elbows flexed at 90 degrees, retracting both scapulae while keeping the entire upper extremity in contact with the wall

3. The subject will horizontally abduct the shoulders above the head while keeping the scapulae retracted and entire upper extremity in contact with wall

4. The subject will then return to the starting position while keeping the scapulae retracted and entire upper extremity in contact with the wall 
Wall Washes
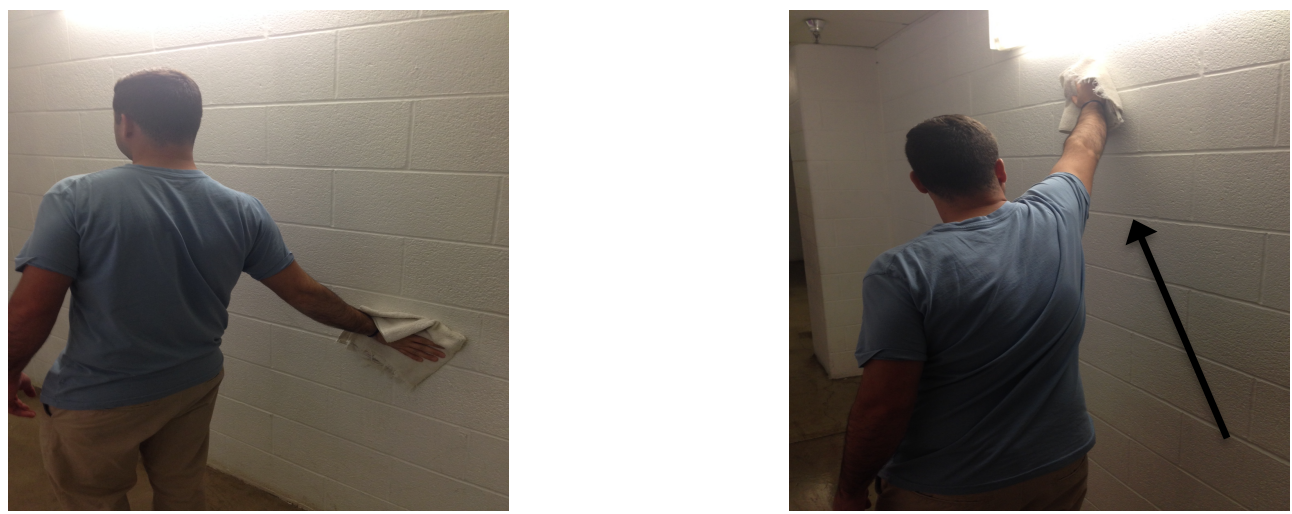

1. The subject will stand next to a wall and place the hand on a towel on the wall

2. The subject's hand should be slightly behind the body

3. The subject should push into the wall to keep the entire hand in contact with the towel

4. While keeping pressure on the towel the subject will slide the towel on a diagonal and then return to the starting position while keeping pressure on the towel

Upright Scapula Punches
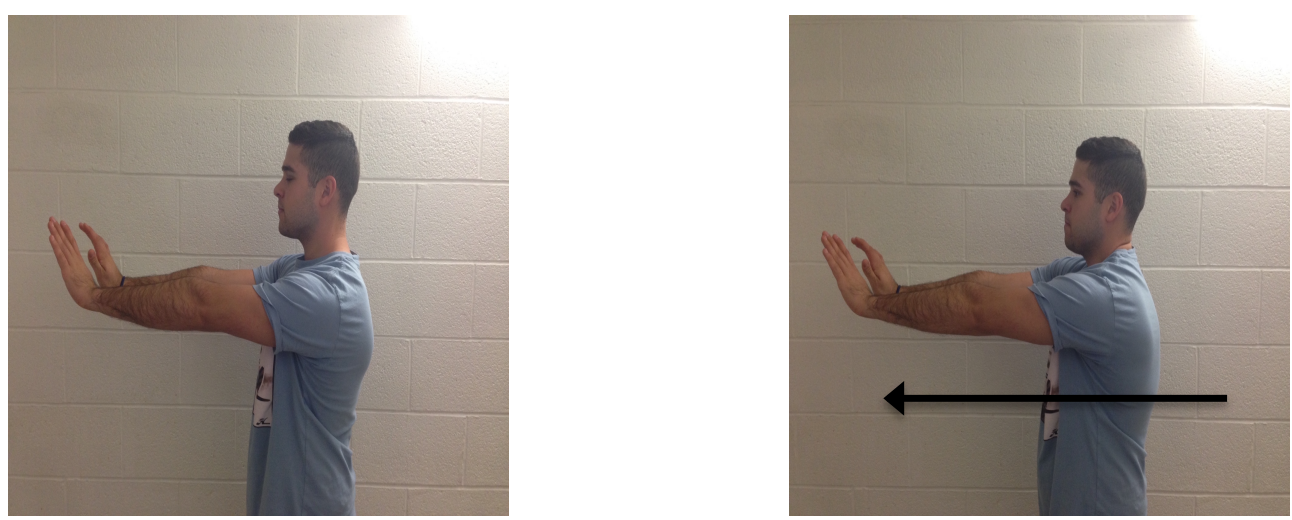

1. The subject will stand erect with both of the shoulders straight out in front with the elbows straight

2. The subject will then move the arms forward without bending the back and hold and then return to the starting position 


\section{Corner Stretch}

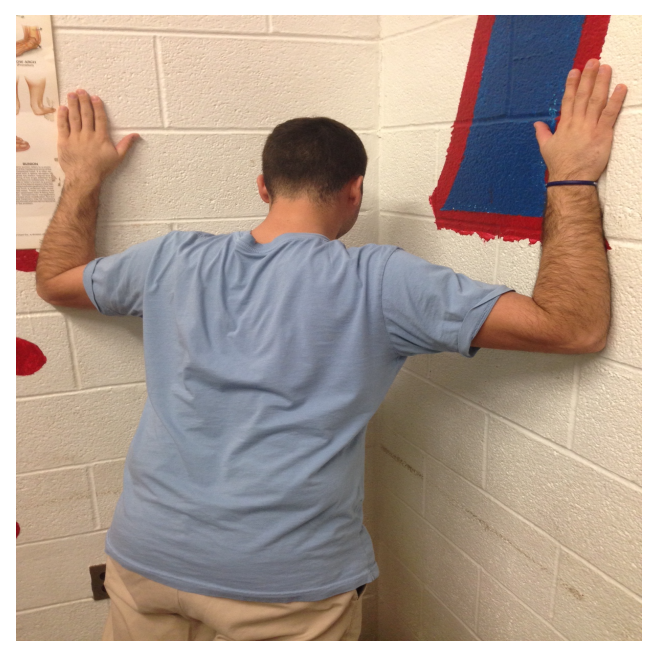

1. The subject will find a corner of a room

2. The subject will place both forearms on the wall, making sure the entire forearm is in contact with the wall

3. The subject will lean forward until sufficient stretch is felt and hold

Static Sternocleidomastoid Stretch

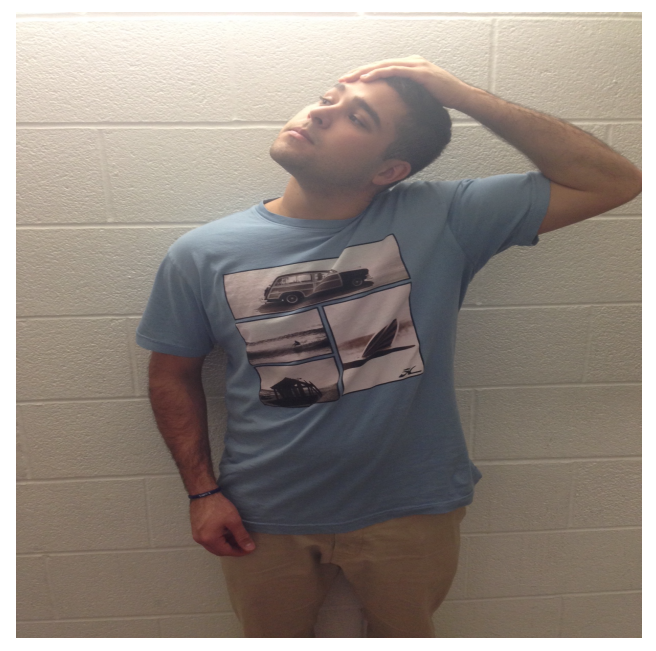

1. The subject will rotate the head as far as it will go to the left then use the right arm to bend the head to the right so that the subject's nose is up in the air and hold

2. The subject will then rotate the head as far as it will go to the right then use the left arm to bend the head to the left so that subject's nose is up in the air and hold 
Y's to I's with chin tuck
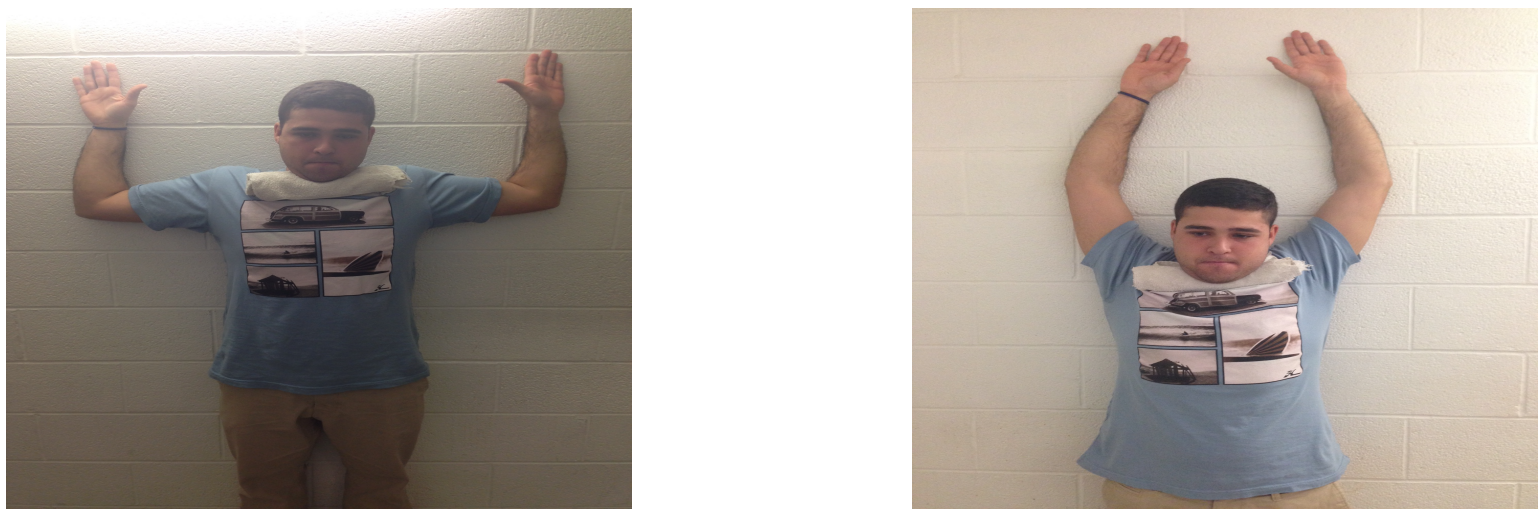

1. The subject will begin with the back against a wall

2. The subject will roll up a towel, place it under the chin, and then tuck the chin to keep it in place

3. The subject will place horizontal abduct the shoulders to 90 degrees and elbows flexed at 90 degrees, retracting both scapulae while keeping the scapulae retracted and entire upper extremity in contact with the wall

4. While keeping their chin tucked the subject will horizontally abduct the shoulders above the head while keeping their scapulae retracted and entire upper extremity in contact with wall

5. The subject will then return to the starting position while keeping the scapulae retracted and entire upper extremity in contact with wall 


\section{Passive Sternocleidomastoid Stretch}

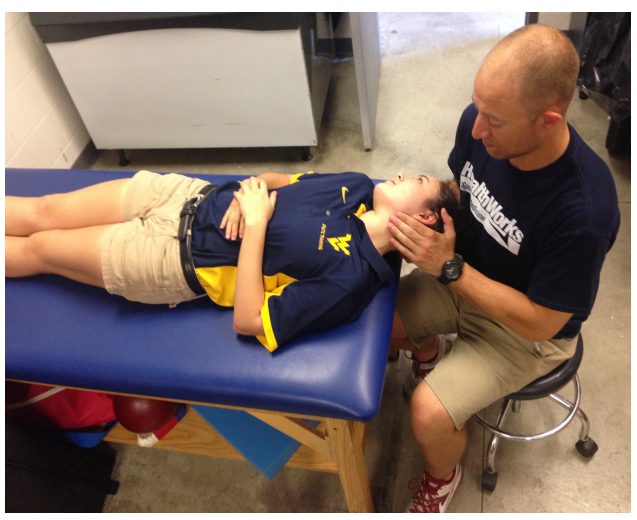

1. The subject will be relaxed in a supine position on the table

2. The clinician will then bring the subjects head into lateral flexion to the left and rotation to the right with one hand and depressing the right shoulder with the other hand and hold

3. The clinician will then bring the subjects head into lateral flexion to the right and rotation to the left with one hand and depressing the left shoulder with the other hand and hold

Passive Pectoralis Minor Stretching

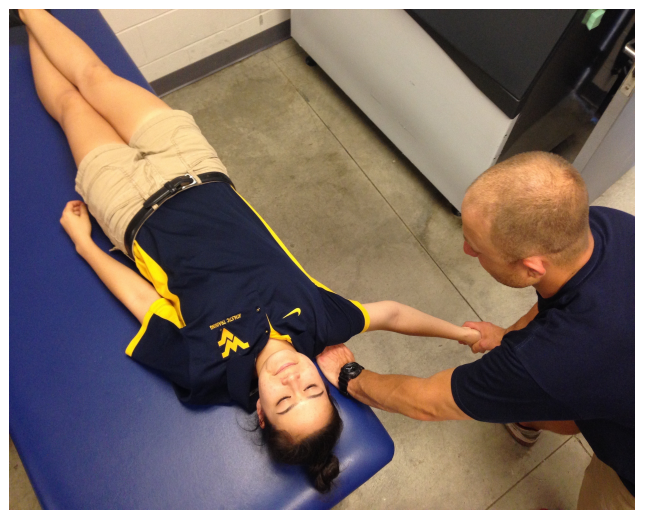

1. The subject will be relaxed in a supine position on the table, with the right shoulder slightly off the table

2. The clinician will bring the subject's right arm into horizontal abduction with one hand while maintaining pressure on the anterior chest wall with the other hand and hold

3. The subject will then be relaxed in a supine position on table, with the right shoulder slightly off the table

4. The clinician will bring the subject's left arm into horizontal abduction with one hand while maintaining pressure on the anterior chest wall with the other hand and hold 


\section{Scapular PNF}

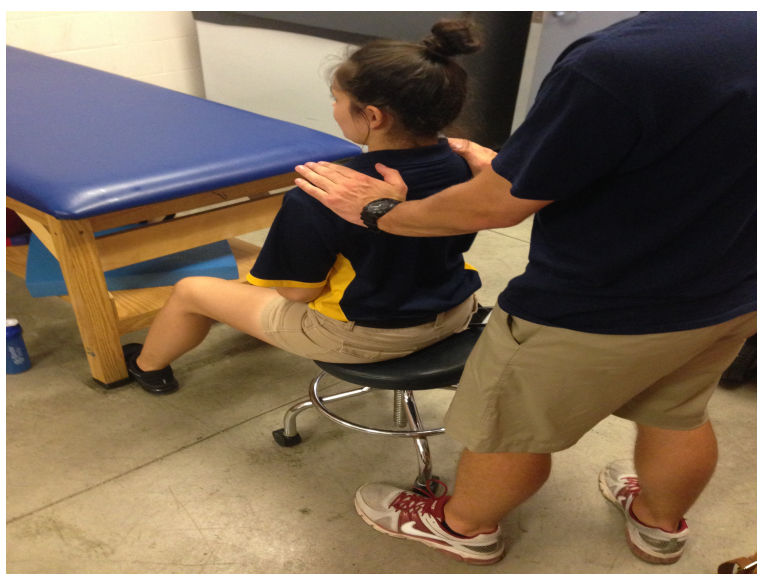

1. The subject will be relaxed and seated in a stool

2. The clinician will give the subject resistance while the subject retracts and depresses both scapulae followed by protraction and elevation of the scapulae

3. This will be done till fatigue

4. The clinician will give the subject resistance while the subject retracts and elevates both scapulae followed by protraction and depression of the scapulae

5. This will be completed until fatigue 

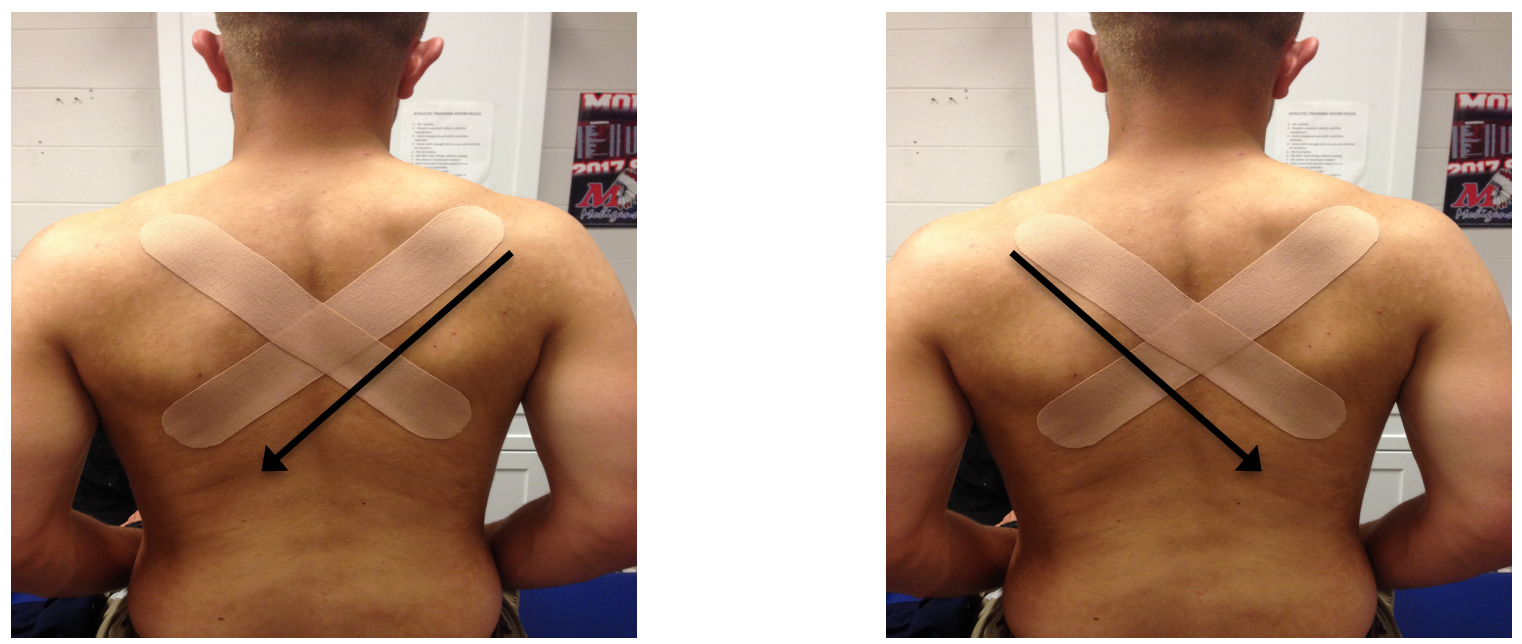

1. Patient will be sitting with shirt removed for males and a sports bra on for females

2. The patient will then retract both scapulae and hold the position

3. The first strip will be placed from the superior border of the right scapula and then pulled down with moderate tension to the inferior scapular angle of the left scapula

4. The second strip will be placed from the superior border of the left scapula and then pulled down with moderate tension to the inferior scapular angle of the right scapula 


\section{APPENDIX D}

\section{ADDITIONAL RESULTS}

Table D1. Baseline CVA, RSA, and Scapular Dyskinesis Score for Subjects $(\mathrm{n}=20)$

\begin{tabular}{llll} 
& Exercise Group & Kinesiotape Group & Significance \\
\hline CVA (deg.) & $47.80 \pm 7.38$ & $48.70 \pm 6.27$ & $\mathrm{p}=.772$ \\
RSA (deg.) & $51.80 \pm 5.94$ & $53.10 \pm 5.86$ & $\mathrm{p}=.628$ \\
Scapular Dyskinesis Score & $4.80 \pm 1.14$ & $4.10 \pm 1.59$ & $\mathrm{p}=.273$ \\
\hline
\end{tabular}

Abbreviations: CVA, craniovertebral angle; RSA, rounded shoulder angle.

Key: No significant difference was found between groups at baseline

Table D2. Pre-test/Post-test Measurements for CVA, RSA, and Scapular Dyskinesis Score

\begin{tabular}{lcccc} 
& \multicolumn{2}{c}{ Exercise Group } & \multicolumn{2}{c}{ Kinesiotape Group } \\
\hline CVA & Pre-test & Post-test & Pre-test & Post-test \\
RSA & $47.80 \pm 7.38$ & $51.70 \pm 5.078$ & $48.70 \pm 6.27$ & $49.50 \pm 9.046$ \\
Scapular Dyskinesis Score & $51.80 \pm 5.94$ & $52.70 \pm 12.841$ & $53.10 \pm 5.86$ & $50.40 \pm 9.058$ \\
\hline & $4.80 \pm 1.14$ & $5.30 \pm .949^{\mathrm{a}}$ & $4.10 \pm 1.59$ & $4.9 \pm 1.101^{\mathrm{a}}$ \\
\hline
\end{tabular}

Abbreviations: CVA, craniovertebral angle; RSA, rounded shoulder angle.

Key: ${ }^{\mathrm{a}}$ significant difference for time

Table D3. Time effect size for CVA, RSA, and Scapular Dyskinesis Score

\begin{tabular}{lcccc} 
& \multicolumn{2}{c}{ Exercise Group } & \multicolumn{2}{c}{ Kinesiotape Group } \\
\hline & Effect Size & Confidence Interval & Effect Size & Confidence Interval \\
\hline CVA & $.53^{\mathrm{b}}$ & -.36 to 1.42 & $.13^{\mathrm{a}}$ & -.75 to 1.00 \\
RSA & $.15^{\mathrm{a}}$ & -.73 to 1.03 & $-.46^{\mathrm{b}}$ & -1.35 to .43 \\
Scapular Dyskinesis Score & $.44^{\mathrm{b}}$ & -.45 to 1.33 & $.50^{\mathrm{b}}$ & -.39 to 1.39 \\
\hline
\end{tabular}

Abbreviations: CVA, craniovertebral angle; RSA, rounded shoulder angle.

Key: ${ }^{a}$ Small effect size. ${ }^{b}$ Moderate effect size. 
Table D4. Group effect size for CVA, RSA, and Scapular Dyskinesis Score

Exercise Group v Kinesiotape Group

\begin{tabular}{lcc}
\hline & Effect Size & Confidence Interval \\
\hline CVA & $.24^{\mathrm{a}}$ & -.64 to 1.12 \\
RSA & $.25^{\mathrm{a}}$ & -.63 to 1.13 \\
Scapular Dyskinesis Score & $.36^{\mathrm{a}}$ & -.52 to 1.25 \\
\hline
\end{tabular}

Abbreviations: CVA, craniovertebral angle; RSA, rounded shoulder angle.

Key: ${ }^{a}$ Small effect size. ${ }^{b}$ Moderate effect size.

Table D5. Mean Time Difference \& MDC Values for CVA, RSA, \& Scapular Dyskinesis Score

\begin{tabular}{lcccc} 
& \multicolumn{2}{c}{ Exercise Group } & \multicolumn{2}{c}{ Kinesiotape Group } \\
\hline & Mean Difference & MDC & Mean Difference & MDC \\
\hline CVA & $3.90^{\mathrm{a}}$ & .755 & $.80^{\mathrm{a}}$ & .755 \\
RSA & .90 & 1.044 & $-2.70^{\mathrm{a}}$ & 1.044 \\
Scapular Dyskinesis Score & $.50^{\mathrm{a}}$ & .494 & $.80^{\mathrm{a}}$ & .494 \\
\hline
\end{tabular}

Abbreviations: CVA, craniovertebral angle; RSA, rounded shoulder angle; MDC, minimal detectable change.

Key: ${ }^{\text {a }}$ Exceeded MDC. 


\section{APPENDIX E}

\section{RECOMMENDATIONS FOR FUTURE RESEARCH}

1. Use an athletic population to determine the generalizable effects of the intervention to athletes.

2. Determine validity of the Hudl app by comparing to a gold standard, 3D kinematic analysis or bone pins to measure FHRSP and scapular dyskinesis.

3. Add sham group to determine if a placebo effect was present by using a regular athletic tape or using KT without any pull.

4. Incorporate an intervention using both exercises that increase the neuromuscular control of the scapular stabilizer and associated musculature and a kinesiotape protocol to determine the efficacy of a concomitant treatment.

5. Increase the duration greater than 4 weeks (i.e. 6,12 , or 18 weeks) of the intervention to determine if the improvements in FHRSP and scapular dyskinesis become significant

6. Use a different $\mathrm{KT}$ protocol with vertical strips that physically cover the $\mathrm{C} 7$ vertebra as well a crossing pattern to cover both acromion processes to determine the effects on FHRSP.

7. Incorporate a sample of subjects with neck and/or shoulder pain to determine the effects of an exercise and KT intervention on a non-healthy population. 


\section{ADDTITIONAL REFERENCES}

41. Yoo W, Yi C, Kim M. Effects of proximity-sensing feedback chair on head, shoulder, and trunk postures when working at a visual display terminal. J Occup Rehabil. 2006;16:631-637.

42. Corrêa, Eliane C.R. and Fausto Bérzin. Efficacy of physical therapy on cervical muscle activity and on body posture in school-age mouth breathing children. Int J Pediatr Otorhi. 2010;71(10):1527-1535.

43. Djordevic OC, Vukicevic D, Katunac L, Jovic S. Mobilization with movement and kinesiotaping compared with a supervised exercises program for painful shoulder: results of a clinical trial. J Manip Physiol Ther. 2012;35(6):454-463.

44. Szeto GP, Straker L, Raine S. A field comparison of neck and shoulder postures in symptomatic and asympomatic office worker. Appl Ergon. 2002;33:75-84.

45. Krabek BJ, Kanarek SL. Cervical spine pain in the competitive athlete. Phys Med Rehabil Clin N Am. 2011;2:1-13.

46. Lee JH, Cynn H, Yi CH, Kwon O, Yoon TL. Predictor variables for forward scapula posture including posterior shoulder tightness. J Bodyw Mov Ther. 2015;19:253-260.

47. Berolo S, Wells RP, Amick BC. Musculoskeletal symptoms among mobile hand-held device users and their relationship to device use: a preliminary study in a Canadian university population. Appl Ergon. 2011;42:371-378.

48. Young JG, Trudeau M, Odell D, Marinelli K, Dennerlein JT. Touch-screen tablet user configurations and case-supported tilt affect head and neck flexion angles. Work. 2012;41:81-91.

49. Lee H, Nicholson LL, Adams RD, Maher CG, Halaki M, Bae SS. Development and psychometric testing of Korean language versions of 4 neck pain and disability questionnaires. Spine. 2006;31:1841-1845.

50. Straker L, Jones KJ, Miller J. A comparison of the postures assumed when using laptop computers and desktop computers. Appl Ergon. 1997;28:263-268.

51. Kibler BW, Sciascia A. Current concepts: scapular dyskinesis. Br J Sport Med. 2010;44:300-305.

52. Yesilyaprak SS, Yuksel E, Kalkan S. Influence of pectoralis minor and upper trapezius lengths on observable scapular dyskinesis. Phys Ther Sport. 2016;19:7-13.

53. Starkey C, Brown SD. Orthopedic and Athletic Injury Examination Handbook. Philadelphia; F. A. Davis Co., 2010. 
54. Burns SA, Cleland JA, Carpenter K, Mintken PE. Interrater reliability of the cervicothoracic and shoulder physical examination in patients with a primary complaint of shoulder pain. Phys Ther Sport. 2016;18:46-55.

55. De Groef A, Van Kampen M, Verloesem N, Clabau E, Christiaens MR, Neven P, Geraerts I, Struyf F, Devoogdt N. Inter-rater reliability of shoulder measurements in middle-aged women. Physio. 2016;7(2):1-24.

56. Oliveira AK, Borges DT, Lins C, Cavalcanti RL, Macedo LB, Brasileiro JS. Immediate effects of Kinesio Taping on Neuromuscular performance of quadriceps and balance in individuals submitted to anterior cruciate ligament reconstruction: a randomized clinical trail. J Sci Med Sport. 2016;19:2-6.

57. Cai C, Au IPH, An W, Cheung RTH. Facilitory and inhibitory effects of Kinesio tape: fact or fad? J Sci Med Sport. 2015;19(2):109-112.

58. Guner S, Alsancak S. Immediate effects of kinesio tape on acute hamstring strain; case report. J Rom Sport Med Soc. 2015;10(1):2305-2308.

59. Kase, K., Wallis, J., Kase, T., 2002a. Clinical Therapuetic Applications of the Kinesio Taping Method. Ken Ikai, Tokyo. 2015. 